\title{
Harmonic Analysis in Discrete Dynamical Systems
}

\author{
Gerardo Pastor ${ }^{1 *}$, Miguel Romera ${ }^{1}$, Amalia Beatriz Orue ${ }^{1}$, Agustin Martin ${ }^{1}$, \\ Marius F. Danca ${ }^{2}$, Fausto Montoya ${ }^{1}$ \\ ${ }^{1}$ Instituto de Seguridad de la Información, CSIC, Madrid, Spain \\ ${ }^{2}$ Romanian Institute of Science and Technology, Cluj-Napoca, Romania \\ Email: *gerardo@iec.csic.es
}

Received February 7, 2012; revised March 9, 2012; accepted March 192012

\begin{abstract}
In this paper we review several contributions made in the field of discrete dynamical systems, inspired by harmonic analysis. Within discrete dynamical systems, we focus exclusively on quadratic maps, both one-dimensional (1D) and two-dimensional (2D), since these maps are the most widely used by experimental scientists. We first review the applications in 1D quadratic maps, in particular the harmonics and antiharmonics introduced by Metropolis, Stein and Stein (MSS). The MSS harmonics of a periodic orbit calculate the symbolic sequences of the period doubling cascade of the orbit. Based on MSS harmonics, Pastor, Romera and Montoya (PRM) introduced the PRM harmonics, which allow to calculate the structure of a $1 \mathrm{D}$ quadratic map. Likewise, we review the applications in 2D quadratic maps. In this case both MSS harmonics and PRM harmonics deal with external arguments instead of with symbolic sequences. Finally, we review pseudoharmonics and pseudoantiharmonics, which enable new interesting applications.
\end{abstract}

Keywords: Harmonic Analysis; Discrete Dynamical Systems; MSS Harmonics; PRM Harmonics; Pseudoharmonics

\section{Introduction}

In this paper we review a branch of harmonic analysis applied to discrete dynamical systems. In general, harmonic analysis has been widely used in experimental applications, as in the field of signal processing. In the same way, harmonic analysis applied to discrete dynamical systems can be a valuable tool for experimental scientists studying nonlinear phenomena. This paper is focused above all in showing some tools with interesting applications in nonlinear phenomena.

At its inception, the harmonic analysis studies the representation of a function as the superposition of basic waves which, in physics, are called harmonics. Fourier analysis and Fourier transforms are the two main branches investigated in this field. The harmonic analysis is soon generalized and in the past two centuries becomes, as noted above, a wide subject with a large number of applications in diverse areas of experimental science.

In order to study nonlinear phenomena, experimental scientists use dynamical systems, whether continuous or discrete. In this review paper we only deal with discrete dynamical systems and more specifically with quadratic maps, above all one-dimensional (1D) quadratic maps and two-dimensional (2D) quadratic maps, which are the most commonly used.

The two most popular 1D quadratic maps are the lo-

"Corresponding author. gistic map $x_{n+1}=\lambda x_{n}\left(1-x_{n}\right)$ and the real Mandelbrot map $x_{n+1}=x_{n}^{2}+c$. The logistic map [1-3] is widely known among experimental scientists studying nonlinear phenomena. Indeed, since Verhulst used it for the first time in 1845 to study population growth [1], it has served to model a large number of phenomena. The real Mandelbrot map is the intersection of the Mandelbrot set [4-6] and the real axis. All the 1D quadratic maps are topologically conjugate [7-9]. Therefore, we can use one of them to study the others.

The most popular 2D quadratic map is, without any doubt, the Mandelbrot set, which is the most representative paradigm of chaos. The Mandelbrot set can be defined as $M=\left\{c \in \mathbf{C}: f_{c}^{k}(0) \rightarrow \infty\right.$ as $\left.k \rightarrow \infty\right\}$, where $f_{c}^{k}(0)$ is the $k$-iteration of the complex polynomial function depending on the parameter $c, f_{c}(z)=z^{2}+c, z$ and $c$ complex, for the initial value $z=0$. In the same way as we use the Mandelbrot set to study the complex case, to study the $1 \mathrm{D}$ case we normally use the real Mandelbrot map [10-12] (likewise we could have used the logistic map), that can be defined again as $M_{r}=\left\{c \in \mathbf{C}: f_{c}^{k}(0) \nrightarrow \infty\right.$ as $\left.k \rightarrow \infty\right\}$, but where now $f_{c}^{k}(0)$ is the $k$-iteration of the $1 \mathrm{D}$ polynomial function depending on the parameter $c, f_{c}(x)=x^{2}+c, x$ and $c$ real, for the initial value $x=0$. In this real Mandelbrot map there are several kinds of points according to the 
multiplier value $\lambda=\left|d\left(f_{c}(x)\right) / \mathrm{d} x\right|_{x=x_{f p}}$ [13]. A similar definition of the multiplier $\lambda$ can be given for the Mandelbrot set complex case, as can be seen for example in [6], where $c$ is defined for the parameter values of the Mandelbrot set, which are complex values, and not only for the real values of the real segment $-2 \leq c \leq 1 / 4$.

In both cases, the real case and the complex one, when $\lambda \neq 1$ one has hyperbolic points. The connected components of the $c$-values set for which $f_{c}^{k}(0)$ converges to a $k$-cycle are periodic hyperbolic components (periodic HCs), or simply HCs [6]. These periodic HCs verify $\lambda<1$, which means they are stable (if $\lambda=0$ they are superstable). A HC is a cardioid or a disc for the complex case (2D hyperbolic components) and a segment for the real case (1D hyperbolic components). Therefore, we can speak indistinctly of periodic orbits (superstable periodic orbit if $\lambda=0$ ) or hyperbolic components, although in $1 \mathrm{D}$ quadratic maps we normally speak of periodic orbits, and in 2D quadratic maps of HCs. The variety of names is due to the fact that this paper is a review of several papers. There are also points where $\lambda>1$ which means they are unstable. These last points are, in addition, preperiodic and they have been later called Misiurewicz points [14-18].

When $\lambda=1$ one has non-hyperbolic points. These points correspond to tangent bifurcation points (or cusp points, where, in the case of a 2D quadratic map, a cardioid-like component is born) and to pitchfork bifurcation points (or root points, where, in the case of a $2 \mathrm{D}$ quadratic map, a disc-like component is born).

Therefore in both cases, 1D and 2D quadratic maps, the two most representative elements are HCs (remember that they are more commonly called periodic orbits in $1 \mathrm{D}$ quadratic maps) and Misiurewicz points. There are many ways to identify both HCs and Misiurewicz points. For example, we can recognize a HC by means of its period, and a Misiurewicz point by its preperiod and period. However, normally a lot of HCs have the same period, and a lot of Misiurewicz points have the same preperiod and period; hence, this way of naming them is not univocal. We are interested in names of HCs and Misiurewicz points that can identify them univocally. When the names identify them univocally, we denominate them identifiers. As we shall see later, in 1D quadratic maps the identifiers we use are the symbolic sequences [19], which are sequences of the type $\mathrm{CX} \cdots \mathrm{X}(\mathrm{X}$ can be a $\mathrm{L}$ for left, or a $\mathrm{R}$ for right). These sequences show the symbolic dynamics of the critical point in the map under consideration. Unfortunately, symbolic sequences can not be used as identifiers in 2D quadratic maps because two different HCs can have the same symbolic sequence. Therefore, to identify a HC (or a Misiurewicz point) in a 2D quadratic map we normally use the external argu- ments (EAs) associated to the external rays of Douady and Hubbard $[15,20,21]$ that land in the cusp/root points of the cardioids/discs (or in the Misiurewicz points). These EAs are given as rational numbers with odd denominator in the case of hyperbolic components, and with even denominator in the case of Misiurewicz points. These rational numbers can also be given as their binary expansions [22], which are the most commonly used, and the only ones used here.

As we shall see later, Metropolis, Stein and Stein (MSS) [23] used a variant of the harmonic analysis within the field of discrete dynamical systems, specifically within a type of $1 \mathrm{D}$ quadratic maps, the logistic map. While in the classical harmonic analysis a function is the superposition of the infinity of its harmonics, the harmonics of MSS (MSS harmonics) of the symbolic sequence (pattern for MSS) of a superstable orbit calculate the symbolic sequences of the period doubling cascade of the original orbit. Therefore, the MSS harmonics are a very valuable tool since, given the symbolic sequence of a superstable orbit (which characterizes the whole HC), the symbolic sequences of the infinity of orbits of its period doubling cascade can easily be calculated. Indeed, if we start from a period- $p$ orbit (or HC), the periods of the orbits calculated are $2 p, 4 p, 8 p, \cdots$ (doubling period cascade, always in the periodic region). In Section 2.1 we shall see in more detail MSS harmonics and, in addition, we also shall see MSS antiharmonics.

Based on MSS harmonics and MSS antiharmonics, Pastor, Romera and Montoya (PRM) introduced in [12] Fourier harmonics (F harmonics) and Fourier antiharmonics (F antiharmonics), which in their subsequent papers were simply called harmonics and antiharmonics in order to avoid confusion within the Fourier analysis. Nevertheless, if we simply call them harmonics, they can be confused with the MSS harmonics. Therefore, in this review we have called them PRM harmonics. While MSS harmonics were introduced by using the logistic map, PRM harmonics were introduced by using the real Mandelbrot map. These PRM harmonics and antiharmonics are a powerful tool that can help us in both the ordering of the periodic orbits of the chaotic region (and not only those of the periodic region as in the case of the MSS harmonics) and the calculation of symbolic sequences of these orbits. As we shall see in more detail in Section 2.2, given the symbolic sequence of a periodic orbit, the PRM harmonics of this orbit are the symbolic sequences of the infinity of last appearance periodic orbits of the chaotic band generated by such an orbit.

As we have just said, in Sections 2.1 and 2.2 we introduce the harmonics/antiharmonics of MSS, and the harmonics/antiharmonics of PRM respectively, in both cases when the identifiers of the periodic orbits are the symbolic sequences corresponding to $1 \mathrm{D}$ quadratic maps. 
In Sections 3.1 and 3.2 we shall see again MSS and PRM harmonics/antiharmonics but now in 2D quadratic maps; that is, when the identifiers are EAs.

When we are working in the chaotic region of $2 \mathrm{D}$ quadratic maps out of the period doubling cascade and out of the chaotic bands, harmonics and antiharmonics have to be generalized. That is what we do in Section 4, where pseudoharmonics and pseudoantiharmonics are introduced. These two new tools will allow new orderings and new calculations in this chaotic region.

\section{Harmonics in 1D Quadratic Maps}

In this section on $1 \mathrm{D}$ quadratic maps we first review the MSS harmonics/antiharmonics, which were introduced by MSS [23]. Given the symbolic sequence of a periodic orbit, MSS harmonics calculate the symbolic sequences of the period doubling cascade of that periodic orbit, placed in the periodic region. Finally, we review the PRM harmonics/antiharmonics [12]. Given the symbolic sequence of a periodic orbit (HC), PRM harmonics calculate the symbolic sequences of the last appearance periodic orbits (or last appearance HCs) of that periodic orbit, placed in the chaotic region. Let us see both cases in Sections 2.1 and 2.2.

\subsection{MSS Harmonics}

The symbolic dynamics is introduced by Morse and Hedlung in 1938 [24]. According to Hao and Zhen [25], based on this theory, Metropolis, Stein and Stein [23] develop the applied symbolic dynamics to the case of one-dimensional unimodal maps, which is simpler and very useful. Applied symbolic dynamics used in the present paper is based on the paper of MSS and on the recipes of Schroeder [26].

The symbolic dynamics is based on the fact that sometimes it is not necessary to know the values of the iteration but it is enough to know if these values are on the left (L) or are on the right (R) of the critical point (C). The sequence of symbols CXX $\cdots X$ (X is an $\mathrm{L}$ for left, or a $\mathrm{R}$ for right) is called symbolic sequence, or pattern.

There are two types of $1 \mathrm{D}$ quadratic maps, rightward maps, $R$-maps, and leftward maps, L-maps [11,12]. The most representative R-map is the logistic map,

$x_{n+1}=\lambda x_{n}\left(1-x_{n}\right)$, and the most representative L-map is the real Mandelbrot map, $x_{n+1}=x_{n}^{2}+c$. In the logistic map the critical point is a maximum, whereas in the real Mandelbrot map the critical point is a minimum. As said before, all the 1D quadratic maps are topologically conjugate [7-9], therefore the logistic map and the real Mandelbrot map have equivalent symbolic dynamics, and the symbolic sequences of one of them can be obtained by interchanging Rs and Ls from the other one.

MSS use the logistic map, an R-map, therefore the
$R$-parity, which is the canonical parity of a R-map, has to be applied. The symbolic sequence of a periodic orbit of a $\mathrm{R}$-map has even R-parity if the number of Rs is even, and it has odd R-parity if the number of Rs is odd [11,12]. Let us see now the definition of harmonic introduced by MSS.

Let $P$ be the pattern of a superstable orbit of the logistic map. The first MSS harmonic of $P, H_{M S S}^{(1)}(P)$, is formed by appending $P$ to itself and changing the second $\mathrm{C}$ to $\mathrm{R}$ (or L) if the R-parity of $P$ is even (or odd). The second MSS harmonic of $P, H_{M S S}^{(2)}(P)$, is formed by appending $H_{M S S}^{(1)}(P)$ to itself and changing the second $\mathrm{C}$ to $\mathrm{R}$ (or $\mathrm{L}$ ) if the R-parity of $H_{M S S}^{(1)}(P)$ is even (or odd). And so on.

The change from $\mathrm{C}$ to $\mathrm{R}$ or $\mathrm{L}$ obeys the relo rule ( $\mathrm{R}$ if Even and L if Odd), which is the canonical rule of a R-map, and a useful mnemonic rule. As mentioned in the introduction, the periods of the successive MSS harmonics of a pattern $P$ of period $p$ are: $2 p, 4 p, 8 p, \cdots$, which correspond to the periods of the patterns of the period doubling cascade of $P$.

\section{Example:}

We start from the period-1 superstable orbit whose pattern is C. To find the patterns of the period doubling cascade of $\mathrm{C}$ we have to obtain the successive MSS harmonics of $\mathrm{C}$ by applying the relo rule. To obtain the first MSS harmonic of $C$ we append $C$ to $C$ (CC) and we change the second $\mathrm{C}$ to $\mathrm{R}$ because the R-parity of $\mathrm{C}$ is even. To obtain the second MSS harmonic of CR we append CR to CR (CRCR) and we change the second C to $\mathrm{L}$ because the R-parity of CR is odd. And so on. The results up to the fifth MSS harmonic, which correspond to the $2^{0}, 2^{1}, 2^{2}, 2^{3}, 2^{4}$ and $2^{5}$ periodic orbits of the period doubling cascade, are:

$$
\begin{aligned}
& H_{M S S}^{(0)}(\mathrm{C})=\mathrm{C}, H_{M S S}^{(1)}(\mathrm{C})=\mathrm{CR}, H_{M S S}^{(2)}(\mathrm{C})=\mathrm{CRLR}, \\
& H_{M S S}^{(3)}(\mathrm{C})=\mathrm{CRLR}^{3} \mathrm{LR}, \\
& H_{M S S}^{(4)}(\mathrm{C})=\mathrm{CRLR}^{3} \mathrm{LRLRLR}^{3} \mathrm{LR}, \\
& H_{M S S}^{(5)}(\mathrm{C})=\mathrm{CRLR}^{3} \mathrm{LRLRLR}^{3} \mathrm{LR}^{3} \mathrm{LR}^{3} \mathrm{LRLRLR}^{3} \mathrm{LR} .
\end{aligned}
$$

Note that, when $i=0, H_{M S S}^{(i)}$ corresponds to the trivial case of the starting point, and only when $i=1,2, \cdots$, $H_{M S S}^{(i)}$ are the first, second, $\cdots$ MSS harmonics, respectively.

Let us see now the definition of antiharmonics, also introduced by MSS.

Let $P$ be the pattern of a superstable orbit of the logistic map. The first MSS antiharmonic of $P, A_{M S S}^{(1)}(P)$, is formed by appending $P$ to itself and changing the second $\mathrm{C}$ to $\mathrm{L}$ (or R) if the R-parity of $P$ is even (or odd). The second MSS antiharmonic of $P, A_{M S S}^{(2)}(P)$, is formed by appending $A_{M S S}^{(1)}(P)$ to itself and changing the second $\mathrm{C}$ to L (or R) if the R-parity of $A_{M S S}^{(1)}(P)$ is even (or odd). And so on.

As can be seen, in this case the mnemonic rule is the lero rule ( $\mathrm{L}$ if Even and $\mathrm{R}$ if Odd), which is the antican- 
onical rule of a R-map. As in the case of the MSS harmonics, the periods of the successive MSS antiharmonics of a pattern $P$ of period $p$ are: $2 p, 4 p, 8 p, \cdots$ Antiharmonics seem to have no interest because they do not correspond to any possible periodic orbit. However, this is not so, as we shall see later.

\section{Example:}

If we start again from the period-1 superstable orbit whose pattern is C, and we calculate up to the third MSS antiharmonic by applying the lero rule, we obtain:

$$
\begin{aligned}
& A_{M S S}^{(0)}(\mathrm{C})=\mathrm{C}, \quad A_{M S S}^{(1)}(\mathrm{C})=\mathrm{CL}, \\
& A_{M S S}^{(2)}(\mathrm{C})=\mathrm{CL}^{3}, \quad A_{M S S}^{(3)}(\mathrm{C})=\mathrm{CL}^{7},
\end{aligned}
$$

that indeed do not correspond to any periodic orbit.

\subsection{PRM Harmonics}

In 1997 the PRM harmonics and PRM antiharmonics were introduced to contribute to the ordering of $1 \mathrm{D}$ quadratic maps [12]. The search of order in chaos, and more specifically in 1D quadratic maps, was early carried out in the well known works of Sharkovsky [27,28]. Sharkovsky's theorem gives a clear ordering of the superstable periodic orbits but only of orbits that appear by the first time. This theorem states that the first appearance of the periodic orbits of the parameter-dependent unimodal maps are in the following universal ordering when the parameter absolute value increases:

$1 \triangleleft 2 \triangleleft 4 \triangleleft 8 \cdots 2^{\mathrm{k}} .9 \triangleleft 2^{\mathrm{k}} .7 \triangleleft 2^{\mathrm{k}} .5 \triangleleft 2^{\mathrm{k}} .3 \cdots 2.9 \triangleleft 2.7$ $\triangleleft 2.5 \triangleleft 2.3 \cdots 9 \triangleleft 7 \triangleleft 5 \triangleleft 3$ where the symbol $\triangleleft$ must be read as "precede to".

The Sharkovsky theorem gives a clear ordering of the first appearance superstable orbits (see Figure 1), but without taking into account either the symbolic sequence or the origin of each periodic orbit. On the contrary, the outstanding work of MSS [23], which also deals with the issue of ordering, uses both the symbolic sequence and the pattern generation; however, it is difficult to see any ordering there (see Figure 2, where we graphically show the MSS superstable periodic orbit generation according to the MSS theorem [23]).

As said before, PRM harmonics were introduced in [12] to better understand the ordering and the generation of the symbolic sequences or patterns of superstable orbits. However, in the same way as the Sharkovsky ordering only treats a part of the total set of the superstable orbits, the first appearance superstable orbits, PRM only treat another part of this set, the last appearance superstable orbits. On the other hand, while MSS or Sharkovsky use the logistic map, a R-map, PRM use the real Mandelbrot map, a L-map, which is the intersection of the Mandelbrot set with the real axis.

From the introduction of PRM harmonics, PRM obtain what they call the harmonic structure of a $1 \mathrm{D}$ quadratic map [12], which results from the generation of all the genes, i.e., the superstable orbits of the period doubling cascade. This harmonic structure obtained from the genes is a way of seeing the ordering that clearly shows the connection between each period doubling cascade component (gene) and the corresponding chaotic band.

One can obtain all the structural patterns by starting out only from the pattern $\mathrm{C}$ of the period-1 superstable orbit. Beginning from this pattern $\mathrm{C}$, all the patterns of the period doubling cascade and the patterns of the last appearance superstable orbits of the chaotic bands are generated. One can clearly see that the origin of each period- $2^{n}$ chaotic band $B_{n}$ is the $n^{\text {th }}$ periodic orbit of the period doubling cascade, with period $2^{n}$, which is the gene $G_{n}$.

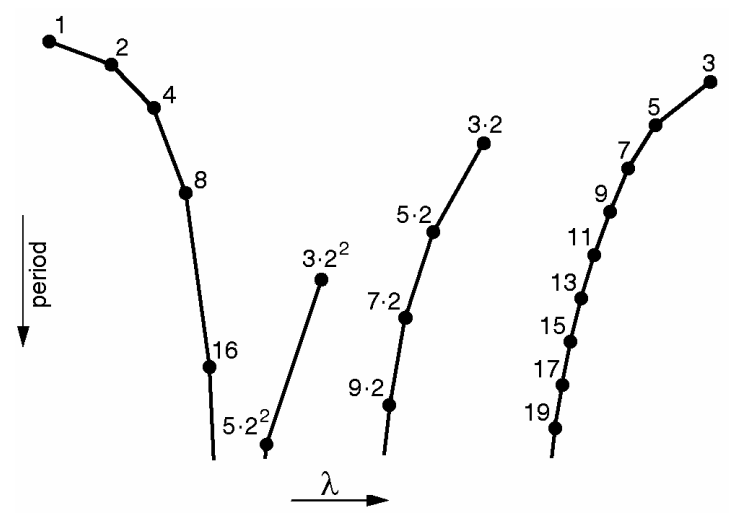

Figure 1. A sketch of the Sharkovsky theorem for the logistic map, $x_{n+1}=\lambda x_{n}\left(1-x_{n}\right)$. First appearance superstable orbits for periods $p \leq 20$ are shown.

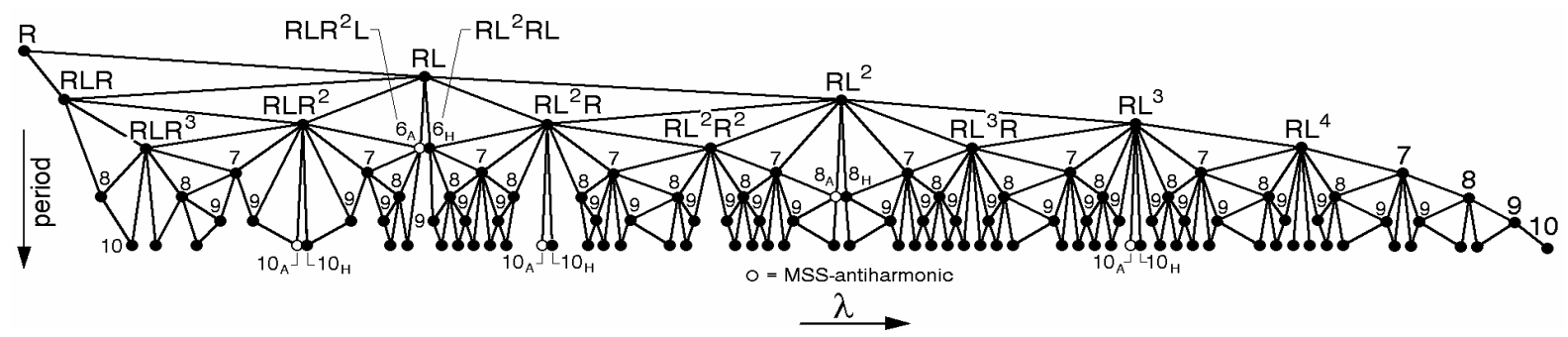

Figure 2. A sketch of the successive application of the Metropolis, Stein, and Stein theorem in the logistic map $x_{n+1}=\lambda x_{n}\left(1-x_{n}\right)$ for $p \leq 10$. Symbolic sequences for periods $p \leq 6$ are shown. 
Let us consider the real Mandelbrot map, which as we have repeated is an L-map. As we know from [12,17], a pattern $P$ has even L-parity if it has an even number of Ls, and has odd L-parity otherwise. L-parity is a concept similar to R-parity, introduced by MSS [23] for the logistic map, a R-map. Now, the definition of PRM harmonics $[12,17]$ can be seen.

Let $P$ be a pattern. The first PRM harmonic of $P$, $H_{P R M}^{(1)}(P)$, is formed by appending $P$ to itself and changing the second $\mathrm{C}$ to $\mathrm{L}$ (or R) if the L-parity of $P$ is even (or odd). The second PRM harmonic of $P$, $H_{P R M}^{(2)}(P)$, is formed by appending $P$ to $H_{P R M}^{(1)}(P)$ and changing the second $\mathrm{C}$ to $\mathrm{L}$ (or $\mathrm{R}$ ) if the L-parity of $H_{P R M}^{(1)}(P)$ is even (or odd), and so on.

As can be seen, in this case the mnemonic rule is the lero rule (L if Even and $\mathrm{R}$ if Odd), which is the canonical rule for an L-map.

Application: The harmonic structure of $1 D$ quadratic maps

Let us see how to generate the chaotic bands in the real Mandelbrot map by beginning just at the origin, i.e., at the period-1 superstable orbit of symbolic sequence $C$. For this purpose, we shall be assisted by Figure 3, where in the upper parts we depict sketches of the PRM harmonics where periods and symbolic sequences are shown and, in the lower parts, we depict the corresponding Mandelbrot set antenna zones by means of the escape lines method [29]. Obviously, since we are in the 1D case, in these lower parts only the intersection of these figures with the real axis make sense, although we use the whole figure in order to better "see" the periodic orbits. Let us note that, in the upper parts, symbolic sequences corresponding to cardioids are only depicted with black half filled circles, while symbolic sequences corresponding to discs are depicted with black circles. In Figure 3(a) we show the PRM harmonics of $\mathrm{C}$ obtained in accordance with the lero rule. To form the first PRM harmonic of $\mathrm{C}$ we add a $\mathrm{C}$ to the $\mathrm{C}$, i.e., we write $\mathrm{CC}$ and we change the second $\mathrm{C}$ into an $\mathrm{L}$, since the L-parity of $\mathrm{C}$ is even. Therefore, the first PRM harmonic of $\mathrm{C}$ is $H_{P R M}^{(1)}(\mathrm{C})=\mathrm{CL}$. To form the second PRM harmonic of $\mathrm{C}$ we add a $\mathrm{C}$ to the first one, i.e., we write CLC and we change the second $\mathrm{C}$ into a $\mathrm{R}$, since the L-parity of $\mathrm{CL}$ is odd, and we obtain $H_{P R M}^{(2)}(\mathrm{C})=\mathrm{CLR}$.

By applying the same procedure, we obtain that the third and fourth PRM harmonics of $\mathrm{C}$ are

$H_{P R M}^{(3)}(\mathrm{C})=\mathrm{CLR}^{2}$ and $H_{P R M}^{(4)}(\mathrm{C})=\mathrm{CLR}^{3}$. As is well known [12,26], CLR, CLR ${ }^{2}, \mathrm{CLR}^{3}, \cdots\left(\mathrm{CRL}, \mathrm{CRL}^{2}\right.$, $\mathrm{CRL}^{3}, \cdots$, in the logistic map) are the symbolic sequences of the last appearance superstable orbits. As it was already shown [12,30], the limit of the PRM harmonics of a pattern can be calculated. In this case the limit $H_{P R M}^{(\infty)}(\mathrm{C})$ is the Misiurewicz point with preperiod 1 and period $1, M_{1,1}$, whose symbolic sequence is (CL)R (if $\mathrm{C}$ is taken into account, the preperiod is 2, and we have $M_{2,1}$, as can be seen in [12]). This point is the tip (C) $[12,30]$, whose parameter value is $c=-2$.

As seen in the figure, we start from the period- 1 superstable orbit $\mathrm{C}$ placed in the periodic region. The first PRM harmonic of $\mathrm{C}$ is the period-2 superstable orbit of the period doubling cascade. All the other PRM harmonics of $\mathrm{C}$ are superstable orbits placed in the period- $2^{0}$ chaotic band $B_{0}$ and are the last appearance superstable orbits of this band. If we consider the period $-2^{0}$ superstable orbit $\mathrm{C}$ as a gene $G_{0}$, then the PRM harmonics of the gene $G_{0}$ generate the period- $2^{0}$ chaotic band $B_{0}$. However, a period- $2^{1}$ superstable orbit placed in the periodic region is also generated. Let us see what happens when this orbit is used as a new gene $G_{1}$.

Let's now look at Figure 3(b) where we show the harmonics of $G_{1}=\mathrm{CL}$. To form its PRM harmonics we add CL to the previous one and we change the second $\mathrm{C}$ into a $\mathrm{L}$ or a $\mathrm{R}$, in accordance with the lero rule. So, we obtain that the first, second, third, and fourth PRM harmonics of $G_{1}$ are

$$
\begin{aligned}
& H_{P R M}^{(1)}\left(G_{1}\right)=\mathrm{CLRL}, H_{P R M}^{(2)}\left(G_{1}\right)=\mathrm{CLRL}^{3}, \\
& H_{P R M}^{(3)}\left(G_{1}\right)=\mathrm{CLRL}^{5}, \text { and } H_{P R M}^{(4)}\left(G_{1}\right)=\mathrm{CLRL}^{7} .
\end{aligned}
$$

The limit of these harmonics is the Misiurewicz point $m_{1}=M_{2,1}=(\mathrm{CLR}) \mathrm{L}$, placed in $c=-1.543689012 \cdots$ $[16,17]$, that separates the period $-2^{0}$ chaotic band $B_{0}$ and the period- $2^{1}$ chaotic band $B_{1}$. The first harmonic generated from CL is the pattern CLRL of period- $2^{2}$, which corresponds to the second superstable orbit of the period doubling cascade of the periodic region of $\mathrm{C}$. All the other PRM harmonics of CL are superstable orbits placed in the period- $2^{1}$ chaotic band $B_{1}$ and, in addition, are the last appearance superstable orbits of this band. Again, if we consider CL as a gene $G_{1}$, we have that the PRM harmonics of the gene $G_{1}$ generate the period- $2^{1}$ chaotic band $B_{1}$ (and a new gene $G_{2}=$ CLRL ).

Let us see now Figure 3(c) where we show the PRM harmonics of the gene $G_{2}=$ CLRL . The first, second, third, and fourth harmonics of $G_{2}$ are

$$
\begin{aligned}
& H_{P R M}^{(1)}\left(G_{2}\right)=\mathrm{CLRL}^{3} \mathrm{RL}, H_{P R M}^{(2)}\left(G_{2}\right)=\mathrm{CLRL}^{3} \overline{\mathrm{RL}}^{3}, \\
& H_{P R M}^{(3)}\left(G_{2}\right)=\mathrm{CLRL}^{3} \overline{\mathrm{RL}}^{5}, \text { and } H_{P R M}^{(4)}\left(G_{2}\right)=\mathrm{CLRL}^{3} \overline{\mathrm{RL}}^{7},
\end{aligned}
$$

the first being a new gene in the periodic region and the others superstable orbits placed in the period $-2^{2}$ chaotic band $B_{2}$. The limit of these harmonics is the Misiurewicz point $m_{2}=M_{4,2}^{(1)}=\left(\mathrm{CLRL}^{2}\right) \mathrm{LR} \quad[16,17]$ which separates the period- $2^{1}$ chaotic band $B_{1}$ and the period- $2^{2}$ chaotic band $B_{2}$. Therefore the PRM harmonics of the gene $G_{2}$ generate the period- $2^{2}$ chaotic band $B_{2}$, and a new gene, $G_{3}=\mathrm{CLRL}^{3} \mathrm{RL}$, of the next chaotic band.

Finally, let us see Figure 3(d), where we show the PRM harmonics of the previous period-8 $\left(2^{3}\right)$ gene 


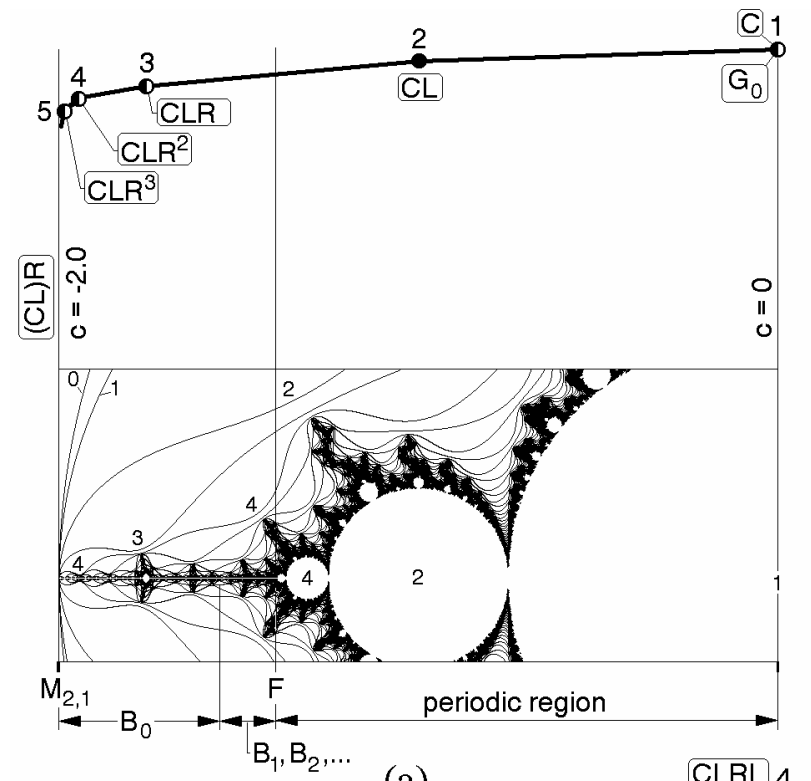

(a)

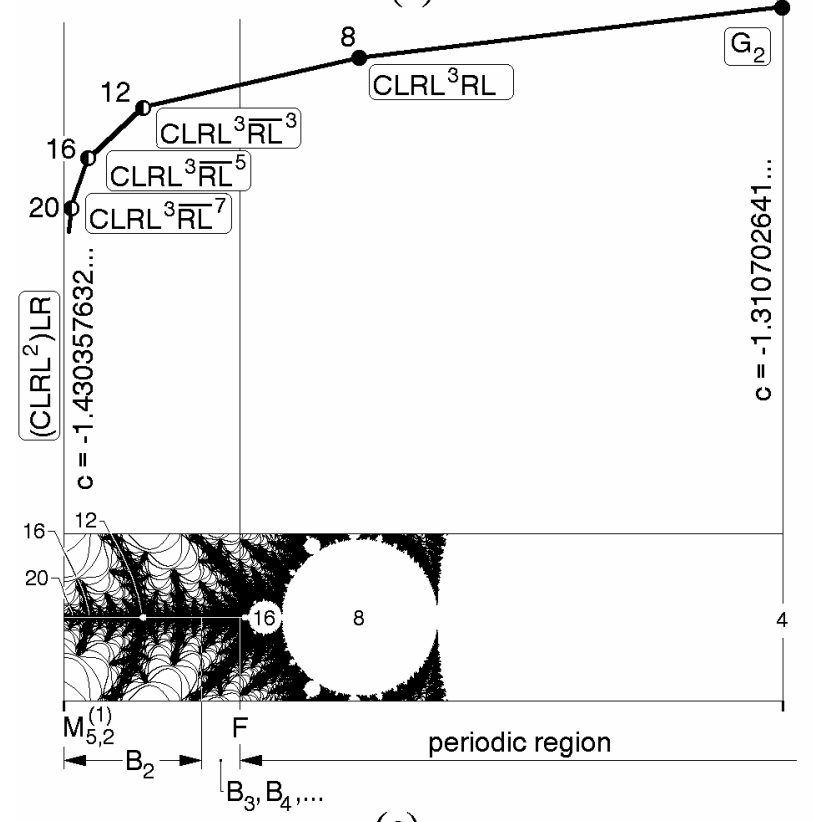

(c)

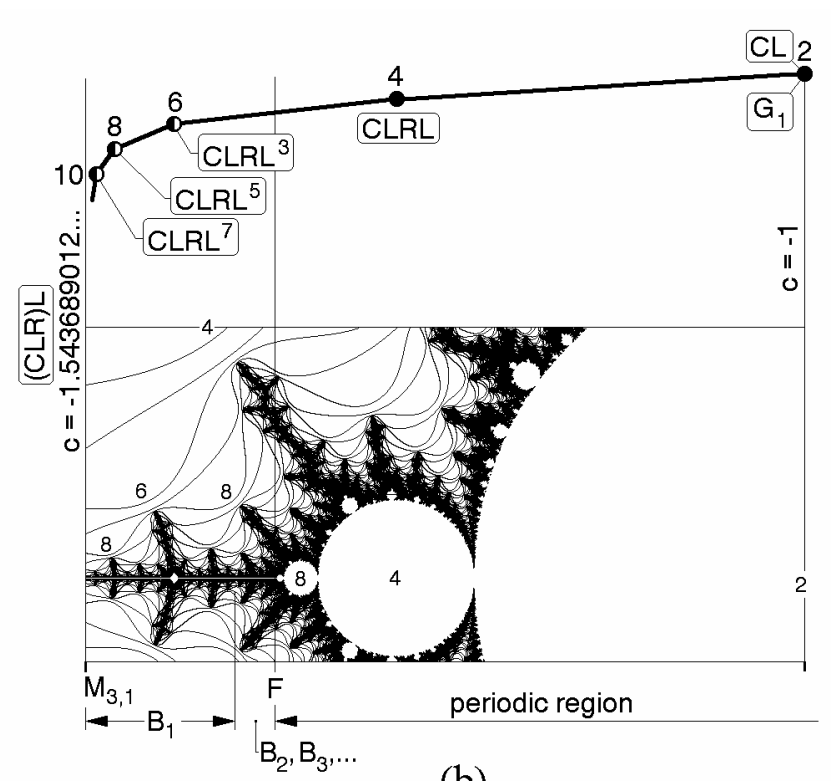

(b)

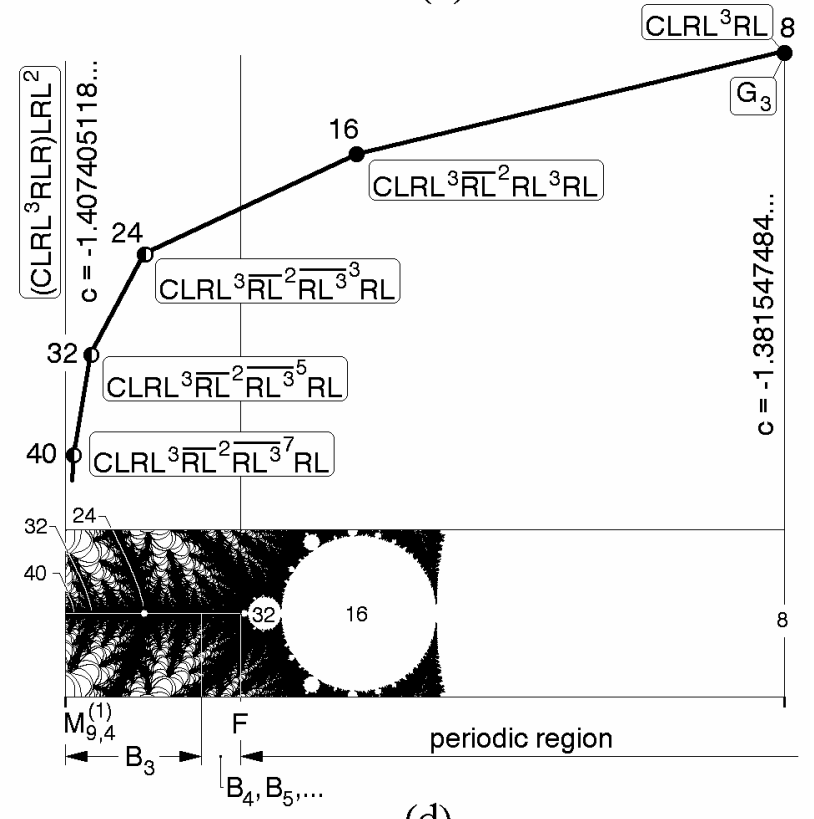

(d)

Figure 3. A sketch of the PRM harmonics of the first four superstable orbits of the period doubling cascade in the real Mandelbrot map. The PRM harmonic generation of last appearance superstable patterns of chaotic bands is shown. (a) Period-1 chaotic band $B_{0}$; (b) period-2 chaotic band $B_{1}$; (c) period-4 chaotic band $B_{2}$; (d) period-8 chaotic band $B_{3}$.

$G_{3}=\mathrm{CLRL}^{3} \mathrm{RL}$. A new period-16 gene in the periodic region, $G_{4}$, and the last appearance superstable orbits of the period- $2^{3}$ chaotic band $B_{3}$ are generated. The limit of these superstable orbits is the Misiurewicz point $m_{3}=M_{8,4}^{(1)}[16,17]$ that separates the period- $2^{2}$ chaotic band $B_{2}$ and the period $-2^{3}$ chaotic band $B_{3}$.

Generalizing, the PRM harmonics of the gene $G_{n}$ generate the last appearance superstable orbits of the period- $2^{n}$ chaotic band $B_{n}$, and a new gene of the period doubling cascade, the gene $G_{n+1}$. Likewise, $H_{P R M}^{(\infty)}\left(G_{n}\right)$ is a Misiurewicz point $m_{n}=M_{2^{n}, 2^{n-1}}$ [10], a primary separator (or band-merging point) of the chaotic bands $B_{n-1}$ and $B_{n}$.

This double procedure (periodic orbits of the period doubling cascade generation and chaotic band generation) continues indefinitely and both, periodic orbits of the period doubling cascade on the right and chaotic bands on the left, meet in the Myrberg-Feigenbaum point [31].

Every pattern of the period doubling cascade is the gene of the corresponding chaotic band. The set of the PRM harmonics of all the genes is what we call the harmonic structure and is schematically shown in Figure 4. 


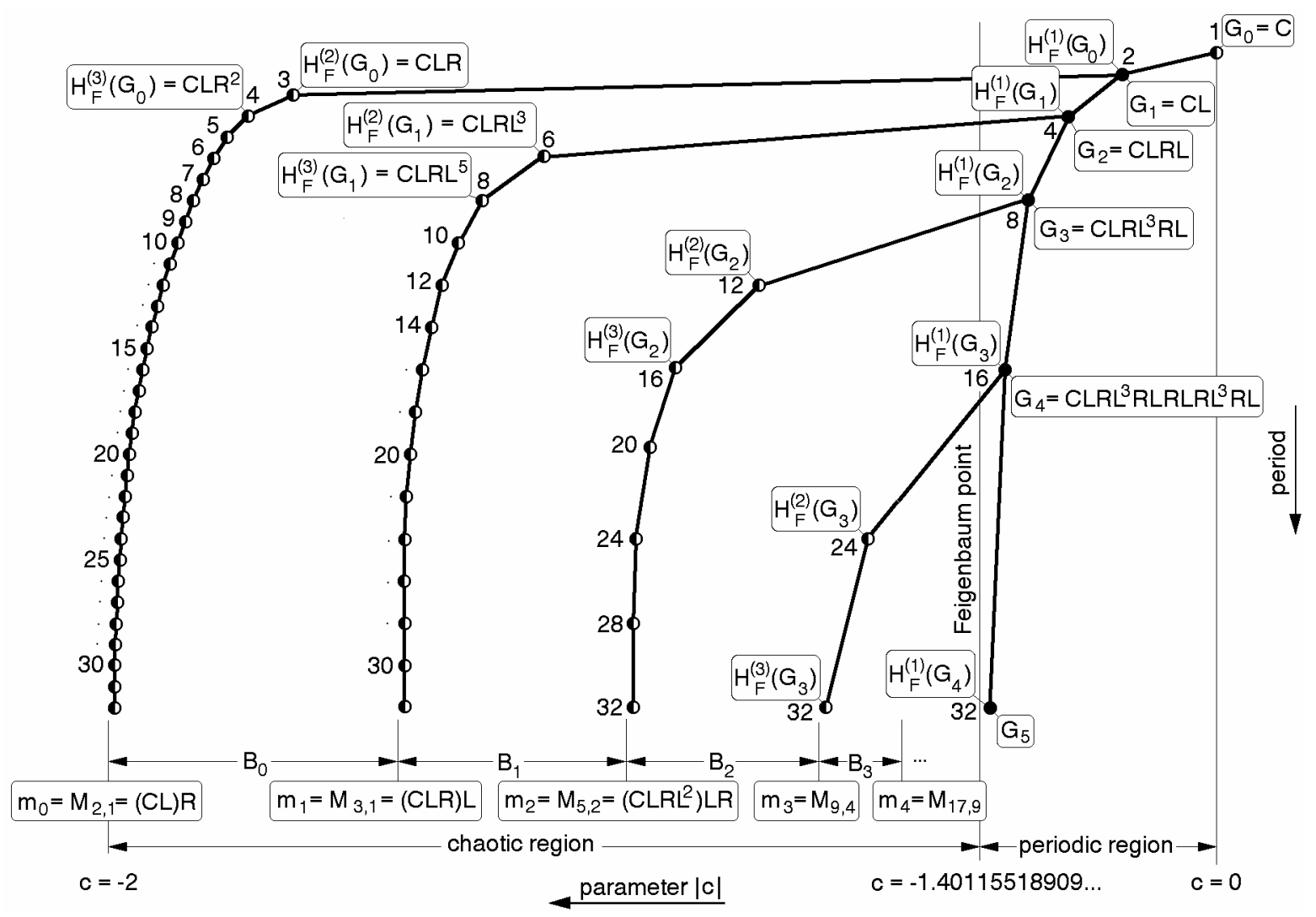

Figure 4. A sketch of the harmonic structure of the real Mandelbrot map. Each period doubling cascade superstable orbit is a gene $G_{n}$. The PRM harmonics of these genes generate the corresponding chaotic band $B_{n}$ and the Misiurewicz point $m_{n}=M_{2^{n} 2^{n-1}}$ that separates the chaotic bands $B_{n-1}$ and $B_{n}$.

The patterns of the harmonic structure are called structural patterns and all of them are PRM harmonics.

In Figure 4 we can see the periodic region and the chaotic region separated by the Myrberg-Feigenbaum point. Likewise, the chaotic region is divided in an infinity of chaotic bands $B_{n}$, separated by Misiurewicz points called separators, $m_{n}, 0 \leq n \leq \infty$. Each structural pattern of each chaotic band and each separator is determined by starting with the only datum of the pattern $\mathrm{C}$, and by applying the successive harmonics to the successive genes, what shows the power as calculation tool of the PRM harmonics.

As can be seen in detail in [12], if we deal with the logistic map, a R-map, instead of with the real Mandelbrot map, a L-map, the result is equivalent to what is shown in Figure 4. However, we have to take into account that in this case, the canonical direction of the logistic map is the rightward direction, the canonical parity is the R-parity and the canonical rule is the relo rule (therefore, we have to interchange Ls and Rs).

To finish this section, we shall see the PRM antiharmonics.

Let $P$ be a pattern. The first PRM antiharmonic of $P$, $A_{P R M}^{(1)}(P)$, is formed by appending $P$ to itself and chang- ing the second $\mathrm{C}$ to $\mathrm{R}$ (or $\mathrm{L}$ ) if the L-parity of $P$ is even (or odd). The second PRM antiharmonic of $P, A_{P R M}^{(2)}(P)$, is formed by appending $P$ to $A_{P R M}^{(1)}(P)$ and changing the second $\mathrm{C}$ to $\mathrm{R}\left(\right.$ or L) if the L-parity of $A_{P R M}^{(1)}(P)$ is even (or odd). And so on.

As in the case of MSS antiharmonics, PRM antiharmonics are also a purely formal construction and never correspond to a periodic orbit either. But, although they have no real existence, they play an important role in some cases, as we shall see later. However, in the case of the structural patterns that we have treated here only PRM harmonics are present.

We are dealing with the real Mandelbrot map, a L-map, and we have to apply the relo rule to obtain antiharmonics. If we were with the logistic map, a R-map, to obtain antiharmonics we would have to apply the lero rule; that is, just the opposite than in the case of harmonics.

As said before, there are two types of $1 \mathrm{D}$ quadratic maps, whose canonical direction are rightward for R-maps and leftward for L-maps. The canonical rule of a R-map is the relo rule, and the canonical rule of a L-map is the lero rule. Harmonics (of MSS or PRM) are obtained by applying the canonical rules, and they grow in the canonical direction. Likewise, antiharmonics (of MSS or PRM) are 
obtained by applying the anticanonical rules, and they grow in the anticanonical direction.

\section{Harmonics in 2D Quadratic Maps}

In the same way as in Section 2 we reviewed harmonics (both MSS harmonics and PRM harmonics) in 1D quadratic maps, in this section we review both types of harmonics in 2D quadratic maps. The main difference between both cases is that the identifiers are the symbolic sequences for the $1 \mathrm{D}$ case, and the EAs (which we only use here in the binary expansion form) for the 2D case.

However, in both cases, the MSS harmonics calculate the identifiers of the period doubling cascade, placed in the periodic region, and the PRM harmonics calculate the identifiers of the last appearance HCs (LAHCs), placed in the chaotic region. As said before, the 2D quadratic map used here is the Mandelbrot set, which can be seen in Figure 5(a). Let us see next MSS harmonics in Section 3.1 and PRM harmonics in Section 3.2.

\subsection{MSS Harmonics}

For the 2D case, when we start from a period- $p$ HC and we progress through its period doubling cascade, we find discs whose periods are $2^{1} \cdot \mathrm{p}, 2^{2} \cdot \mathrm{p}, 2^{3} \cdot \mathrm{p}, \cdots$ In the same way as MSS introduced the concept of harmonics in 1D unimodal maps [23], by extension we call MSS harmonics of a HC of the Mandelbrot set to the set constituted by itself and all the discs of its period-doubling cascade (obviously we refer to their identifier).

Let $\left(. \bar{a}_{1}, \bar{a}_{2}\right)$ be the two EAs of a HC. By taking into account that the EAs of the period-2 disc are $(.01, .10)$ (see Figure 5(a)), it is easy to obtain the EAs of a MSS harmonic of $\left(\bar{a}_{1}, \bar{a}_{2}\right)$ from the tuning algorithm of Douady and Hubbard [21,32]. Therefore, we can define the MSS harmonics as follows:

Let $\left(. \bar{a}_{1}, \bar{a}_{2}\right)$ be the two EAs of a HC. The successive MSS harmonics of the HC are given by:

$$
\begin{aligned}
H_{M S S}^{(0)}\left(. \bar{a}_{1}, . \bar{a}_{2}\right)= & \left(. \bar{a}_{1}, \bar{a}_{2}\right), H_{M S S}^{(1)}\left(\overline{a_{1}}, \overline{a_{2}}\right)=\left(\overline{a_{1} a_{2}}, \overline{a_{2} a_{1}}\right), \quad \begin{array}{l}
\text { can be calculated. Calculating the MSS harmonics, up to } \\
\text { the third, we obtain: }
\end{array} \\
& H_{M S S}^{(0)}(\overline{.00111}, \overline{.01000})=(\overline{.00111}, \overline{.01000}), \quad H_{M S S}^{(1)}(\overline{.00111}, \overline{.01000})=(\overline{.0011101000}, \overline{0100000111}), \\
& H_{M S S}^{(2)}(\overline{.00111}, \overline{.01000})=(\overline{.00111010000100000111}, \overline{.01000001110011101000}), \\
& H_{M S S}^{(3)}(\overline{.00111}, \overline{.01000})=(\overline{.0011101000010000011101000001110011101000},), \cdots,
\end{aligned}
$$

as can be seen in Figure 6(b).

Antiharmonics of MSS seem again to have no interest, as we can deduce next from their definition:

Let $\left(. \bar{a}_{1}, \bar{a}_{2}\right)$ be the two EAs of a HC. The successsive MSS antiharmonics of the HC are given by:

$$
A_{M S S}^{(0)}\left(. \bar{a}_{1}, \bar{a}_{2}\right)=\left(. \bar{a}_{1}, \bar{a}_{2}\right), \quad A_{M S S}^{(1)}\left(. \bar{a}_{1}, . \bar{a}_{2}\right)=\left(\overline{a_{1} a_{1}}, . \overline{a_{2} a_{2}}\right) \text {, }
$$

$H_{M S S}^{(2)}\left(\overline{a_{1}}, \overline{a_{2}}\right)=\left(\overline{a_{1} a_{2} a_{2} a_{1}}, \overline{a_{2} a_{1} a_{1} a_{2}}\right)$,

$H_{M S S}^{(3)}\left(\overline{a_{1}}, \overline{a_{2}}\right)=\left(\overline{a_{1} a_{2} a_{2} a_{1} a_{2} a_{1} a_{1} a_{2}}, \overline{a_{2} a_{1} a_{1} a_{2} a_{1} a_{2} a_{2} a_{1}}\right), \ldots$

$H_{M S S}^{(\infty)}\left(. \bar{a}_{1}, . \bar{a}_{2}\right)$ are the EAs of the Myrberg-Feigenbaum point. This notable point has neither binary nor rational EAs.

\section{Example 1}

The EAs of the Mandelbrot set main cardioid are $(\overline{0}, \overline{1})$. By applying the previous expressions, the EAs of the successive discs of the period doubling cascade of such a main cardioid can be calculated. The first MSS harmonics, up to the fourth, are:

$$
\begin{aligned}
& H_{M S S}^{(0)}(.0, .1)=(.0, .1), H_{M S S}^{(1)}(. \overline{0}, \overline{1})=(. \overline{01}, \overline{10}), \\
& H_{M S S}^{(2)}(. \overline{0}, \overline{1})=(\overline{0110}, \overline{1001}), \\
& H_{M S S}^{(3)}(\overline{0}, \overline{1})=(\overline{01101001}, \overline{10010110}) \text { and } \\
& H_{M S S}^{(4)}(. \overline{0}, \overline{1})=(\overline{0110100110010110}, \overline{1001011001101001}),
\end{aligned}
$$

as can be seen in Figure 5(a).

\section{Example 2}

If we start from the cardioid of any other midget, we can also calculate the EAs of the discs of its period doubling cascade. Look at Figure 5 where Figure 5(a) shows the Mandelbrot set; Figure 5(b) shows a sketch of the shrub (1/3) marked with the rectangle c in Figure 5(a); and Figure 5(c) shows the shrub (1/3) that is a magnifycation of the mentioned rectangle c. Let $(\overline{.00111}, \overline{01000})$ be the EAs of the period-5 cardioid placed in the branch 11 of the shrub (1/3) [33] (see firstly Figures 5(b) and 5(c)). Figure 6(a) shows a magnification of the branch 11 where this period-5 representative can be better observed, and Figure 6(b) shows an additional magnification of such a period-5 representative. The EAs of the successive discs of the period doubling cascade of such a period-5 cardioid
$A_{M S S}^{(2)}\left(\bar{a}_{1}, \bar{a}_{2}\right)=\left(\overline{a_{1} a_{1} a_{1} a_{1}}, \overline{a_{2} a_{2} a_{2} a_{2}}\right), \cdots$

Therefore, all of them are the same, which is the starting HC.

\subsection{PRM Harmonics}

Let us see now the PRM harmonics of a HC in this 2D case. 


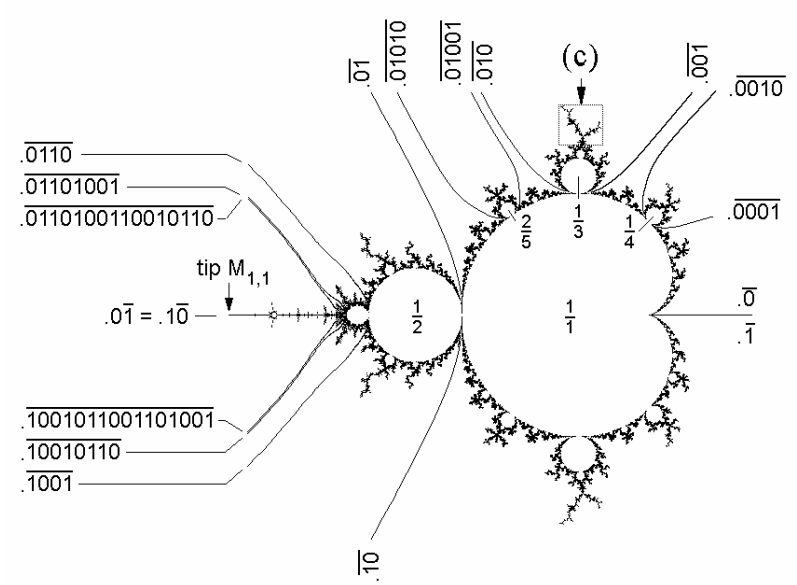

(a)

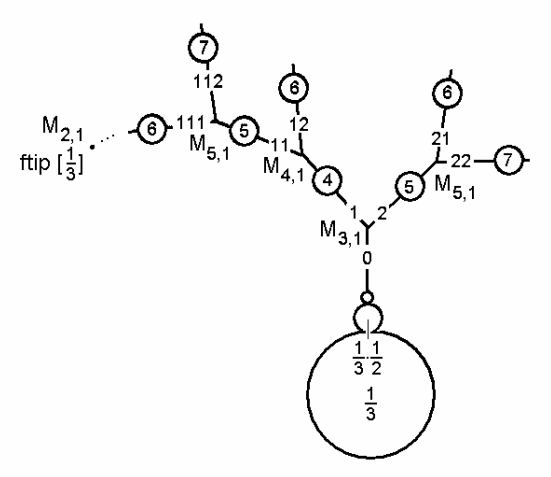

(b)

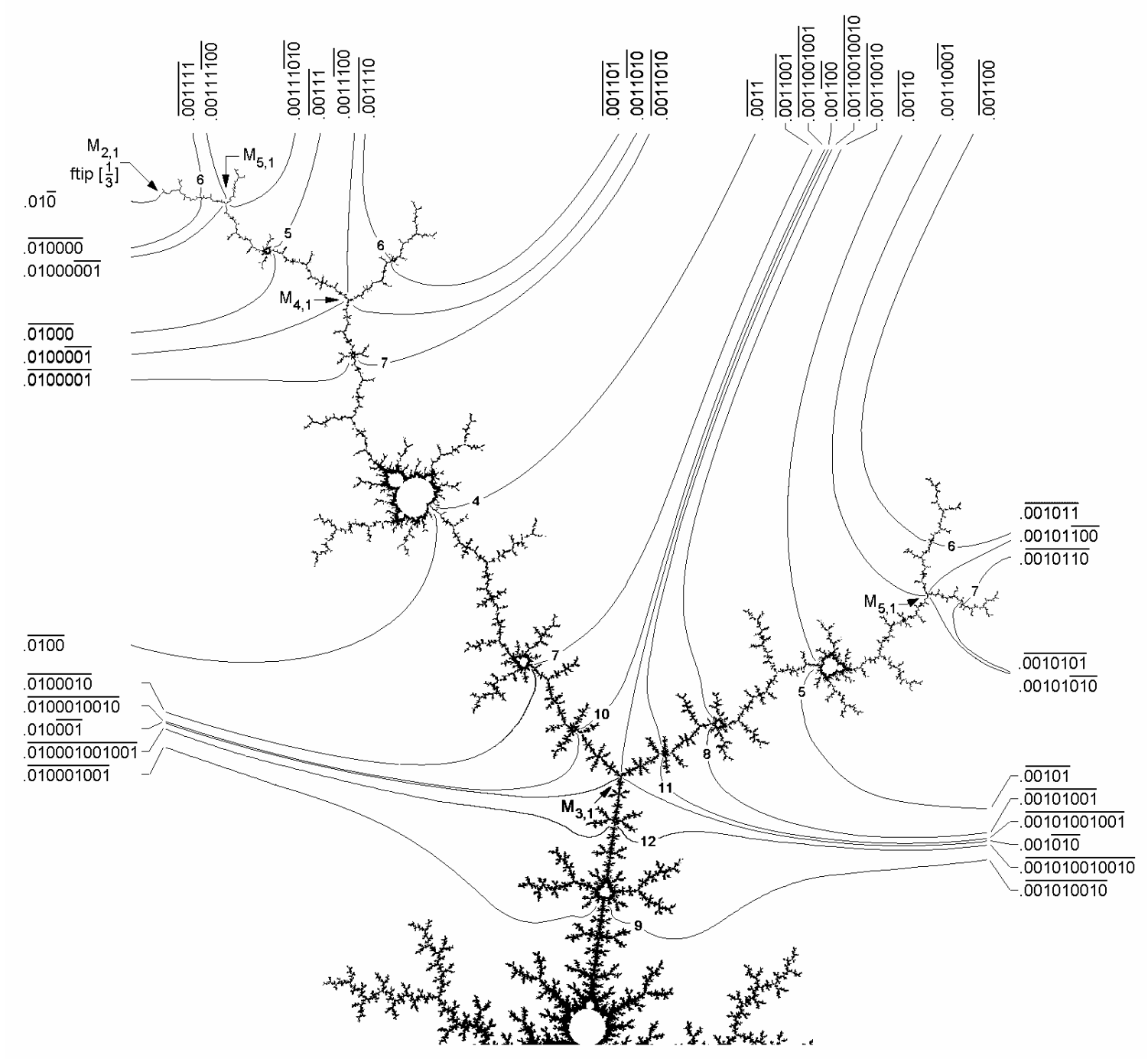

(c)

Figure 5. (a) Mandelbrot set with the first four external arguments of the period doubling cascade, and other significant external arguments. Shrub (1/3) is framed in rectangle c. (b) Sketch of the shrub (1/3) corresponding to the rectangle c marked in (a). (c) Shrub (1/3) that is a magnification of the rectangle c marked in (a). 


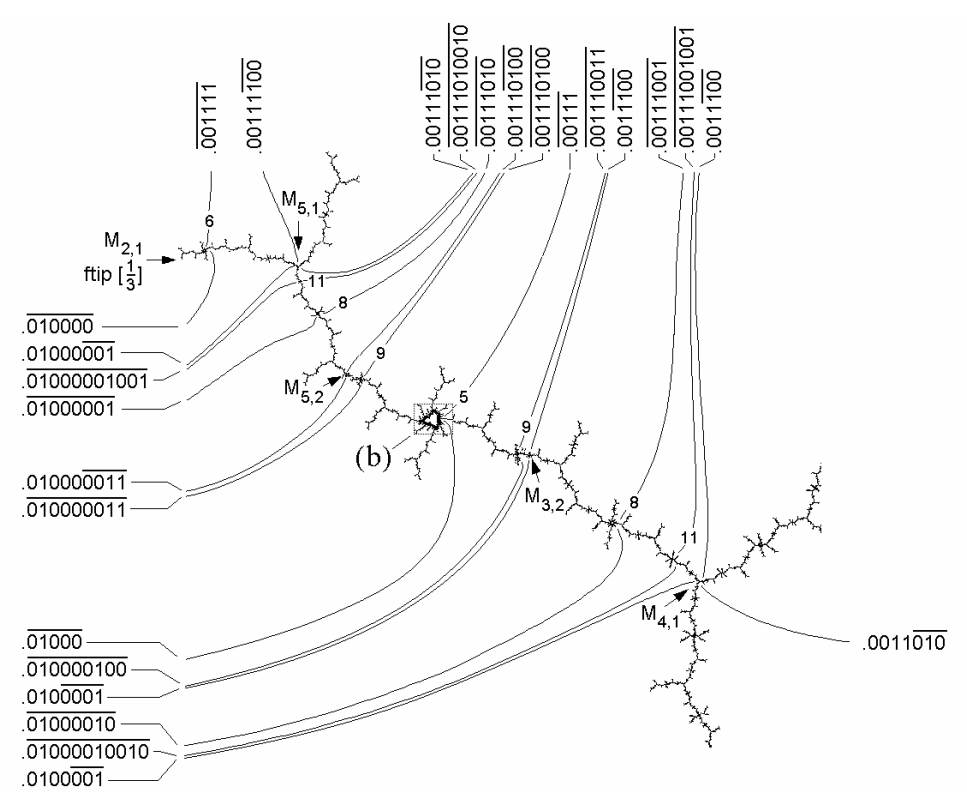

(a)

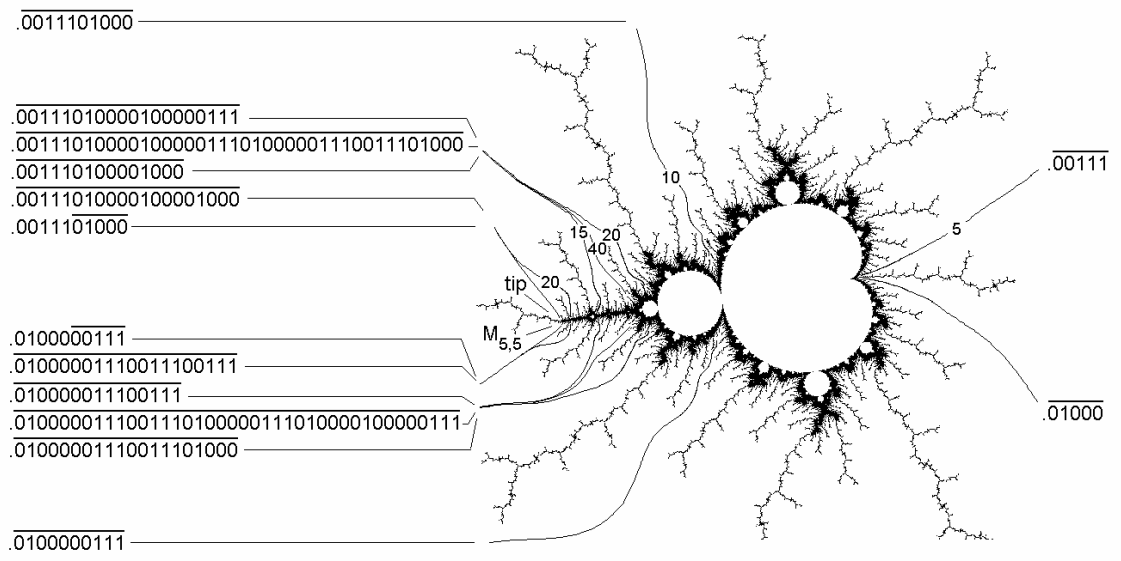

(b)

Figure 6. (a) Magnification of the branch 11 of the shrub (1/3) shown in Figures 5 (b) and (c), where the period-5 representative can be better observed. (b) Magnification of such a period-5 representative shown in the rectangle $b$ marked in (a), with some of its important external arguments.

Let $\left(. \bar{a}_{1}, \bar{a}_{2}\right)$ be the EAs of a HC given in form of binary expansion [22]. The EAs of the order $i$ PRM harmonics of $\left(. \bar{a}_{1}, \bar{a}_{2}\right)$ are given by:

$$
H_{P R M}^{(i)}\left(. \bar{a}_{1}, \bar{a}_{2}\right)=\left(\overline{a_{1} \underbrace{a_{2} a_{2} \cdots a_{2}}_{i}}, \overline{a_{2} \underbrace{a_{1} a_{1} \cdots a_{1}}_{i}}\right) .
$$

When $i=0,1,2,3, \cdots$, Equation (1) calculates a sequence of HCs. When $i \rightarrow \infty$, Equation (1) becomes:

$H_{P R M}^{(\infty)}\left(. \bar{a}_{1}, \bar{a}_{2}\right)=\left(\overline{a_{1} \underbrace{a_{2} a_{2} \cdots a_{2}}_{\infty}}, \overline{a_{2} \underbrace{a_{1} a_{1} \cdots a_{1}}_{\infty}}\right)=\left(. a_{1} \bar{a}_{2}, . a_{2} \bar{a}_{1}\right)$

two preperiodic arguments, and therefore Misiurewicz points.
In the $1 \mathrm{D}$ case, we obtained the harmonic structure through repeated application of the PRM harmonics.

Similarly, in the 2D case Equation (1), applied to a given $\mathrm{HC}$ when $i=0,1,2,3, \cdots$, calculates a sequence of HCs which are the LAHCs of the corresponding chaotic band of the original HC. Indeed, let us analyze, as an example, the main cardioid that we consider as a gene $G_{0}$ (see Figure 7). By applying Equation (1) we obtain the successive PRM harmonics of $G_{0}$. For $i=0$ we obtain $G_{0}$, a trivial case that normally not will be taken into account, for $i=1$ we obtain a new gene $G_{1}$ in the periodic region, and for $i \geq 2$ we obtain the LAHCs of the chaotic band $B_{0}$. If we apply now Equation (2) to $G_{0}$, we obtain the Misiurewicz point $M_{1,1}$, which is the upper extreme of the chaotic band $B_{0}$. That is, $G_{0}$ can be 


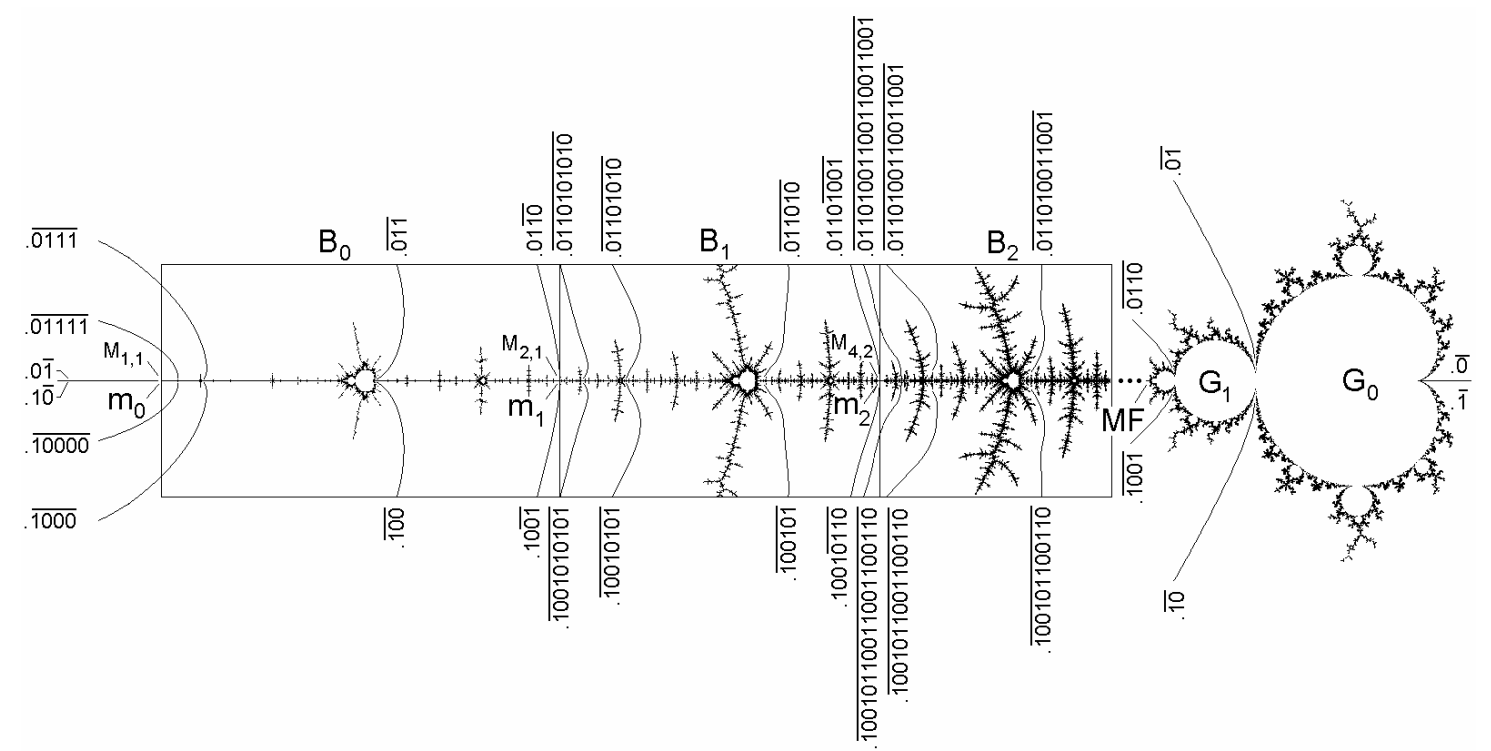

Figure 7. The neighbourhood of the main antenna of the Mandelbrot set with three chaotic bands, $B_{0}, B_{1}$ and $B_{2}$, showing the EAs of their first LAHCs. Likewise, the main cardioid $G_{0}$ and the discs of the period doubling cascade, $G_{1}, G_{2}, \cdots$, showing their EAs.

considered the gene or generator of the chaotic band $B_{0}$. Likewise, by applying now Equation (1) to the new gene $G_{1}$, we obtain, after the trivial case of $i=0$, first a new gene $G_{2}$ in the periodic region, and then the LAHCs of the chaotic band $B_{1}$. If we apply now Equation (2) to $G_{1}$, we obtain the Misiurewicz point $M_{2,1}$, which is the upper extreme of the chaotic band $B_{1}$. That is, $G_{1}$ can be considered the gene or generator of the chaotic band $B_{1}$. In general, by applying Equation (1) to a gene $G_{n}$ we obtain, after the trivial case, first a new gene $G_{n+1}$ in the periodic region, and then the LAHCs of the chaotic band $B_{n}$. If we apply now Equation (2) to $G_{n}$, we obtain the Misiurewicz point $M_{2^{n} \cdot 2^{n-1}}$, which is the upper extreme of the chaotic band ${ }^{2^{n}} \dot{2}_{n}^{2^{n-1}}$. That is, $G_{n}$ can be considered the gene or generator of the chaotic band $B_{n}$.

What we have just seen is similar to the harmonic structure of the 1D case. However, since now we are in the 2D case, we only calculate the structure of the chaotic bands of the cardioid considered. Let us see two examples, the first one applied to the main cardioid (see Figure 7), and the second one to the period-5 midget of the branch 11 of the shrub (1/3) in the chaotic region (see Figure 6(b)).

\section{Example 1}

In Figure 7 we can see the neighbourhood of the main antenna of the Mandelbrot set with three chaotic bands, $B_{0}, B_{1}$ and $B_{2}$, and also the main cardioid $G_{0}$ and the discs of the period doubling cascade, $G_{1}, G_{2}, \cdots$. The EAs of the period-1 main cardioid, the gene $G_{0}$, are $G_{0}=(. \overline{0}, . \overline{1})$. If Equation (1) is applied to this main cardioid, $H_{P R M}^{(i)}(. \overline{0}, . \overline{1})$, for $i=0,1,2,3, \cdots$, the sequence
$(. \overline{0}, \overline{1}),(\overline{01}, \overline{10}),(\overline{.011}, \overline{100})$, $(\overline{.0111}, \overline{1000}),(\overline{.01111}, \overline{10000}), \cdots$

is obtained. The values of this sequence (without taken into account the first one) are: first the gene $G_{1}$, and then the LAHCs of the chaotic band $B_{0}$. If now we apply Equation (2), we obtain $H_{P R M}^{(\infty)}(. \overline{0}, . \overline{1})=(.0 \overline{1}, .1 \overline{0})$, a Misiurewicz point, $m_{0}=M_{1,1}$, which is the upper extreme of $B_{0}$. Likewise, let us consider now $H_{P R M}^{(1)}(. \overline{01}, \overline{10})=(\overline{.01}, \overline{10})$ as a new gene $G_{1}=(\overline{.01}, \overline{10})$. By applying Equation (1) to $G_{1}, H_{P R M}^{(i)}(\overline{.01}, . \overline{10})$ for $i=0,1,2,3, \cdots$, we obtain the sequence

$(\overline{.01}, \overline{10}),(\overline{.0110}, \overline{1001}),(\overline{.011010}, \overline{100101})$,

$(\overline{.01101010}, \overline{10010101})$,

$(\overline{.0110101010}, \overline{1001010101}), \cdots$,

which corresponds to (after the obvious $G_{1}$ ) first the gene $G_{2}$, and then the LAHCs of the chaotic band $B_{1}$. If now we apply Equation (2) we obtain $H_{P R M}^{(\infty)}(. \overline{01}, \overline{10})=(.01 \overline{0}, .1001)$, a Misiurewicz point, $m_{1}=M_{2,1}$, which is the upper extreme of $B_{1}$. Finally, we can consider $H_{P R M}^{(1)}(\overline{.01}, . \overline{10})=(\overline{.0110}, . \overline{1001})$ as a new gene $G_{2}=(\overline{.0110}, \overline{1001})$. By applying Equation (1) to $G_{2}, \quad H_{P R M}^{(i)}(\overline{0110}, \overline{1001})$ for $i=0,1,2,3, \cdots$, we obtain the sequence 
$(\overline{.0110}, \overline{1001}),(\overline{.01101001}, \overline{10010110})$,

$(\overline{.011010011001}, \overline{100101100110})$,

$(\overline{.0110100110011001}, \overline{1001011001100110})$,

$(\overline{.01101001100110011001}, \overline{10010110011001100110}) \cdots$.

After $G_{2}$, these terms are first the gene $G_{3}$, and then the LAHCs of the chaotic band $B_{2}$. By applying Equation (2) we obtain

$$
H_{P R M}^{(\infty)}(\overline{.0110}, \overline{1001})=(.01101001, .10010110),
$$

a Misiurewicz point, $m_{2}=M_{4,2}$, which is the upper extreme of $B_{2}$. And so on, such that, in a general case, $G_{n}$ generates the chaotic band $B_{n}$ (not shown in the figure).

\section{Example 2}

Let us see the period- 5 midget of the branch 11 of the $\operatorname{shrub}(1 / 3)$ shown in Figure 6(b) which is a magnification of the rectangle b shown in Figure 6(a). Let us consider this period-5 cardioid as a gene whose EAs are

$$
G_{0}=(\overline{.00111}, \overline{.01000}) \text {. }
$$

If we apply Equation (1),

$H_{P R M}^{(i)}\left(G_{0}\right)=H_{P R M}^{(i)}(\overline{.00111}, \overline{.01000})$, for $i=0,1,2,3, \cdots$, we obtain the sequence

$(\overline{.00111}, \overline{01000}),(\overline{.0011101000}, \overline{.0100000111})$,

$(\overline{.001110100001000}, \overline{010000011100111})$ ，

$(\overline{.00111010000100001000}, \overline{01000001110011100111}), \cdots$,

whose terms (for $i \geq 2$ ) are the LAHCs of the chaotic band $B_{0}$. If now we apply Equation (2),

$$
H_{P R M}^{(\infty)}(\overline{.00111}, \overline{.01000})=(.00111 \overline{01000}, .01000 \overline{00111}) \text {, }
$$

a Misiurewicz point, $m_{0}=M_{5,5}$, which is both the tip of the midget and the upper extreme of $B_{0}$. As always, we can do the same procedure in order to calculate the LAHCs of the rest of the chaotic bands. We have not done it to avoid problems with the figure.

Antiharmonics of PRM neither seem to have any interest, as in previous cases. Indeed, according to their definition:

Let $\left(. \bar{a}_{1}, \bar{a}_{2}\right)$ be the EAs of a HC given in form of binary expansions [22]. The EAs of the order $i$ PRM antiharmonics of $\left(. \bar{a}_{1}, \bar{a}_{2}\right)$ are given by:

$$
A_{P R M}^{(i)}\left(. \bar{a}_{1}, \bar{a}_{2}\right)=(\overline{a_{1} \underbrace{a_{1} a_{1} \cdots a_{1}}_{i}}, \overline{a_{2}} \underbrace{a_{2} a_{2} \cdots a_{2}}_{i})=\left(. \bar{a}_{1}, \bar{a}_{2}\right)
$$

When $i \rightarrow \infty$, Equation (3) becomes:

$$
A^{(\infty)}\left(. \bar{a}_{1}, \bar{a}_{2}\right)=(\overline{a_{1} \underbrace{a_{1} a_{1} \cdots a_{1}}_{\infty}}, \overline{a_{2}} \underbrace{a_{2} a_{2} \cdots a_{2}}_{\infty})=\left(. \bar{a}_{1}, . \bar{a}_{2}\right)(4)
$$

The result in both equations is the starting HC. However, these concepts will be very useful in the next section.

\section{Pseudoharmonics and Pseudoantiharmonics in the 2D Case}

We have just seen that the PRM harmonics are a powerful tool for calculating some EAs in 2D quadratic maps. However, we can go much further if we introduce an extension of these calculation tools, which we simply call pseudoharmonics and pseudoantiharmonics [35]. These new tools are applied to the EAs of two HCs, as we can see next.

\subsection{Introduction of Pseudoharmonics and Pseudoantiharmonics}

The introduction of pseudoharmonics and pseudoantiharmonics can be seen in detail in [35]. Let us first introduce pseudoharmonics. Let $\left(\overline{a_{1}}, \overline{a_{2}}\right)$ be the external arguments of a HC, and let $\left(\bar{b}_{1}, \overline{b_{2}}\right)$ be the external arguments of other HC that is related with the first one, as will be seen later. The external arguments of the order $i$ pseudoharmonics of $\left(. \overline{a_{1}}, \overline{a_{2}}\right)$ and $\left(\overline{b_{1}}, \overline{b_{2}}\right)$ are:

$$
P H^{(i)}\left[\left(\overline{a_{1}}, \overline{a_{2}}\right) ;\left(\overline{b_{1}}, \overline{b_{2}}\right)\right]=(\overline{a_{1} \underbrace{b_{2} b_{2} \cdots b_{2}}_{i}}, \overline{a_{2}} \underbrace{b_{1} b_{1} \cdots b_{1}}_{i})
$$

When $i=0,1,2,3, \cdots$, Equation (5) calculates a sequence of HCs that we shall determine afterwards. When $i \rightarrow \infty$, Equation (5) becomes:

$$
\begin{aligned}
P H^{(\infty)}\left[\left(\overline{a_{1}}, \overline{a_{2}}\right) ;\left(\overline{b_{1}}, \overline{b_{2}}\right)\right] & =(\overline{a_{1} \underbrace{b_{2} b_{2} \cdots b_{2}}_{\infty}}, \overline{a_{2}} \underbrace{b_{1} b_{1} \cdots b_{1}}_{\infty}) \\
& =\left(. a_{1} \overline{b_{2}}, . a_{2} \overline{b_{1}}\right)
\end{aligned}
$$

Equation (6) calculates a pair of external arguments of a Misiurewicz point. Later we shall analyze this result in every possible case.

Let us now introduce pseudoantiharmonics. Let $\left(\overline{a_{1}}, \overline{a_{2}}\right)$ be the external arguments of a HC, and let $\left(\overline{b_{1}}, \overline{b_{2}}\right)$ be the external arguments of other HC, that is related with the first one. The external arguments of the order $i$ pseudoantiharmonics of $\left(. \bar{a}_{1}, \bar{a}_{2}\right)$ and $\left(\overline{b_{1}}, \overline{b_{2}}\right)$ are: 


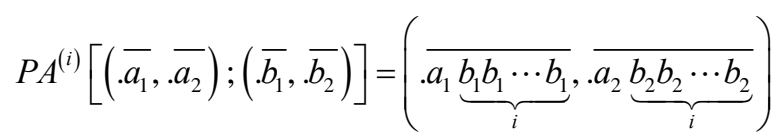

When $i=0,1,2,3, \cdots$, Equation (7) calculates a sequence of HCs, and not the starting $\mathrm{HC}$ as in the case of Equation (3). Therefore, although PRM antiharmonics seemed to be of no use, they have been useful in order to introduce pseudoantiharmonics. When $i \rightarrow \infty$, Equation (7) becomes:

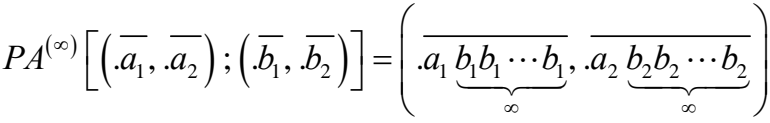

$$
\begin{aligned}
& =\left(. a_{1} \overline{b_{1}}, . a_{2} \overline{b_{2}}\right)
\end{aligned}
$$

Equation (8) calculates a pair of EAs of a Misiurewicz point, and not the starting $\mathrm{HC}$ as in the case of Equation (4). Again PRM antiharmonics are suitable to introduce pseudoantiharmonics that, as we shall see, are very useful to calculate more EAs.

Pseudoharmonics and pseudoantiharmonics are, as has been said, a generalization of PRM harmonics and PRM antiharmonics in the 2D case; therefore, these two last ones have to be a particular case of the two first ones. Indeed, when $\left(\overline{b_{1}}, \overline{b_{2}}\right)=\left(\overline{a_{1}}, \overline{a_{2}}\right)$, Equations (5), (6), (7) and (8) become Equations (1), (2), (3) and (4). Thus, in this case, pseudoharmonics and pseudoantiharmonics have become PRM harmonics and PRM antiharmonics. Let us see it from other approach. Pseudoharmonics and pseudoantiharmonics are applied to two HCs whereas PRM harmonics and PRM antiharmonics are applied to only one HC. However, PRM harmonics and PRM antiharmonics can be thought as pseudoharmonics and pseudoantiharmonics where their two HCs are the same.

\subsection{Some Considerations to Calculate Pseudoharmonics and Pseudoantiharmonics}

To apply what we have seen so far, we shall briefly remember some concepts that can be seen in detail in [35].

We call a descendant [35] of $\left(\overline{a_{1}}, \overline{a_{2}}\right)$ and $\left(\overline{b_{1}}, \overline{b_{2}}\right)$ to any of the HCs obtained from Equations (5) and (7). Likewise, the zone occupied by all the descendants calculated by using these equations is the zone of descendants [35]. In other to calculate a descendant, given the first pair, $\left(\overline{a_{1}}, \overline{a_{2}}\right)$, the second pair cannot be any $\left(\overline{b_{1}}, \overline{b_{2}}\right)$. Indeed, $\left(\overline{b_{1}}, \overline{b_{2}}\right)$ has to be an ancestor [35] of $\left(\overline{a_{1}}, \overline{a_{2}}\right)$.

Let us briefly see the ancestors of $\left(\overline{a_{1}}, \overline{a_{2}}\right)$, which will be used in the calculation of pseudoharmonics and pseu- doantiharmonics (descendants). The second pair $\left(\overline{b_{1}}, \overline{b_{2}}\right)$ is an ancestor of the first pair $\left(\overline{a_{1}}, \overline{a_{2}}\right)$ if $\left(\overline{a_{1}}, \overline{a_{2}}\right)$ is a descendant of $\left(\overline{b_{1}}, \overline{b_{2}}\right)$ and $\left(. \overline{c_{1}}, \overline{c_{2}}\right)$, where $\left(\overline{c_{1}}, \overline{c_{2}}\right)$ itself is an ancestor of $\left(\overline{b_{1}}, \overline{b_{2}}\right)$. Note that any $\mathrm{HC}$ is an ancestor of itself. Note also that the ancestor of a HC has lesser or equal period than the period of such a HC. In order to determine the ancestors of $\left(\overline{a_{1}}, \overline{a_{2}}\right)$, next we shall remember some points.

As known from $[33,36]$, the shrub of the $n$-ary hyperbolic component $\frac{1}{1} \cdot \frac{q_{1}}{p_{1}} \cdots \frac{q_{N}}{p_{N}}$, shrub $\left(\frac{1}{1} \cdot \frac{q_{1}}{p_{1}} \cdots \frac{q_{N}}{p_{N}}\right)$, has $n$ subshrubs or chaotic bands, $S_{1} \cdots S_{N}$, each one of them generated by one HC called gene or generator $[33,35,36]$ of the chaotic band or subshrub. The generator of the last subshrub, $S_{N}$, is the main cardioid $\frac{1}{1}$, that is the first component of the generation route of $\frac{1}{1} \cdot \frac{q_{1}}{p_{1}} \cdots \frac{q_{N}}{p_{N}}$. The generator of the last but one subshrub, $S_{N-1}$, is the primary disc $\frac{1}{1} \cdot \frac{q_{1}}{p_{1}}$, that is the second component of the generation route of $\frac{1}{1} \cdot \frac{q_{1}}{p_{1}} \cdots \frac{q_{N}}{p_{N}}$. Likewise, the generator of $S_{N-2}$, is the secondary disc $\frac{1}{1} \cdot \frac{q_{1}}{p_{1}} \cdot \frac{q_{2}}{p_{2}}$, that is the third component of the generation route of $\frac{1}{1} \cdot \frac{q_{1}}{p_{1}} \cdots \frac{q_{N}}{p_{N}}$, and so on. Finally, the generator of the first subshrub, $S_{1}$, is the $(N-1)$-ary disc $\frac{1}{1} \cdot \frac{q_{1}}{p_{1}} \cdots \frac{q_{N-1}}{p_{N-1}}$, that is the $N^{\text {th }}$ component of the generation route of $\frac{1}{1} \cdot \frac{q_{1}}{p_{1}} \cdots \frac{q_{N}}{p_{N}}$. Hence, in general, the generator of the subshrub $S_{i}$ is the $(N-i)$ ary disc $\frac{1}{1} \cdot \frac{q_{1}}{p_{1}} \cdots \frac{q_{N-i}}{p_{N-i}}$, that is the $(N-i+1)^{\text {th }}$ component of the generation route of $\frac{1}{1} \cdot \frac{q_{1}}{p_{1}} \cdots \frac{q_{N}}{p_{N}}$.

Let $\left(\overline{a_{1}}, \overline{a_{2}}\right)$ be the representative of a branch (whose branch associated number is $d_{1} d_{2} \cdots d_{m}$ [33]) of $S_{i}$. The first ancestor of $\left(\bar{a}_{1}, \overline{a_{2}}\right)$ is the generator $\frac{1}{1} \cdot \frac{q_{1}}{p_{1}} \cdots \frac{q_{N-i}}{p_{N-i}}$ of the chaotic band or subshrub $S_{i}$ where the branch $d_{1} d_{2} \cdots d_{m}$ is found. The second, third, $\cdots$ ancestors of $\left(\overline{a_{1}}, \overline{a_{2}}\right)$ are: $\frac{1}{1} \cdot \frac{q_{1}}{p_{1}} \cdots \frac{q_{N-i+1}}{p_{N-i+1}}$, $\frac{1}{1} \cdot \frac{q_{1}}{p_{1}} \cdots \frac{q_{N-i+2}}{p_{N-i+2}}, \cdots$, in the generation route of 
$\frac{1}{1} \cdot \frac{q_{1}}{p_{1}} \cdots \frac{q_{N}}{p_{N}}$, up to $\frac{1}{1} \cdot \frac{q_{1}}{p_{1}} \cdots \frac{q_{N-1}}{p_{N-1}}$ and $\frac{1}{1} \cdot \frac{q_{1}}{p_{1}} \cdots \frac{q_{N}}{p_{N}}$, all of them in the periodic region. The following ancestors of $\left(\overline{a_{1}}, \overline{a_{2}}\right)$, now in the chaotic region, are the representatives of the branches $d_{1}, d_{1} d_{2}, \cdots, d_{1} d_{2} \cdots d_{m}$. As can be seen, all the ancestors of the chaotic region are in $S_{i}$, and there is no one in the other subshrubs. What we call ancestor route includes all the ordered ancestors of $\left(\overline{a_{1}}, \overline{a_{2}}\right)$ from the generator $\frac{1}{1} \cdot \frac{q_{1}}{p_{1}} \cdots \frac{q_{N-i}}{p_{N-i}}$ to

$\frac{1}{1} \cdot \frac{q_{1}}{p_{1}} \cdots \frac{q_{N}}{p_{N}}$ in the periodic region, followed by the representatives of the branches $d_{1}, d_{1} d_{2}, \cdots, d_{1} d_{2} \cdots d_{m}$ in the chaotic region.

\subsection{Zones of Descendants}

As can be seen in detail in [35], depending on the ancestor $\left(\overline{b_{1}}, \overline{b_{2}}\right)$ we shall have a different zone of descendants. Let us see the most important cases.

$1^{\text {st }}$ case: $\left(\overline{b_{1}}, \overline{b_{2}}\right)$ is the first ancestor

If $\left(\overline{a_{1}}, \overline{a_{2}}\right)$ is the representative of the branch $d_{1} d_{2} \cdots d_{m}$ of $S_{i}$, the first ancestor of $\left(\overline{a_{1}}, \overline{a_{2}}\right)$, $\left(\bar{b}_{1}, \overline{b_{2}}\right)$, is the generator $\frac{1}{1} \cdot \frac{q_{1}}{p_{1}} \cdots \frac{q_{N-i}}{p_{N-i}}$ of $S_{i}$ [35]. In this case, $P H^{(i)}\left[\left(\overline{a_{1}}, \overline{a_{2}}\right) ;\left(\overline{b_{1}}, \overline{b_{2}}\right)\right]$ calculates the representatives of the branches $d_{1} d_{2} \cdots d_{m} \underbrace{1 \cdots 1}_{i}$. That is to say, it successively calculates first the representative of the branch $d_{1} d_{2} \cdots d_{m}$, and then the representatives of the branches $d_{1} d_{2} \cdots d_{m} 1, d_{1} d_{2} \cdots d_{m} 11$, and so on. Obviously, when $i \rightarrow \infty$ one has:

$$
P H^{(\infty)}\left[\left(\overline{a_{1}}, \overline{a_{2}}\right) ;\left(\overline{b_{1}}, \overline{b_{2}}\right)\right]=\left(. a_{1} \overline{b_{2}}, . a_{2} \overline{b_{1}}\right)
$$

that is the pair of external arguments of the Misiurewicz point placed in the upper extreme of the branches $d_{1} d_{2} \cdots d_{m} 11 \cdots$. Therefore,

$$
P H^{(\infty)}\left[\left(\overline{a_{1}}, \overline{a_{2}}\right) ;\left(\overline{b_{1}}, \overline{b_{2}}\right)\right]=\left(. a_{1} \overline{b_{2}}, . a_{2} \overline{b_{1}}\right)
$$

calculates the upper extreme of the zone of descendants of $\left(\overline{a_{1}}, \overline{a_{2}}\right)$ and $\left(\overline{b_{1}}, \overline{b_{2}}\right)$, and $P H^{(i)}\left[\left(\overline{a_{1}}, \overline{a_{2}}\right) ;\left(\overline{b_{1}}, \overline{b_{2}}\right)\right]$ calculates de LAHCs in that zone. The zone of descendants finishes in a tip or upper extreme of $S_{i}$; therefore, this zone of descendants covers the whole subshrub $S_{i}$.

$2^{\text {nd }}$ case: $\left(\overline{b_{1}}, \overline{b_{2}}\right)$ is the second ancestor,

$$
\frac{1}{1} \cdot \frac{q_{1}}{p_{1}} \cdots \frac{q_{N-i+1}}{p_{N-i+1}}
$$

Let $\left(\overline{a_{1}}, \overline{a_{2}}\right)$ be the representative of a branch $d_{1} d_{2} \cdots d_{m}$ of $S_{i}$. Since the first ancestor is $\frac{1}{1} \cdot \frac{q_{1}}{p_{1}} \cdots \frac{q_{N-i}}{p_{N-i}}$, that is the generator of $S_{i}$, the second ancestor is $\frac{1}{1} \cdot \frac{q_{1}}{p_{1}} \cdots \frac{q_{N-i+1}}{p_{N-i+1}}$. Let $\left(\overline{b_{1}}, \overline{b_{2}}\right)$ be the external arguments of this second ancestor. Then

$$
\begin{aligned}
& P H^{(\infty)}\left[\left(\overline{a_{1}}, \overline{a_{2}}\right) ;\left(\overline{b_{1}}, \overline{b_{2}}\right)\right] \text { and } \\
& P A^{(\infty)}\left[\left(\overline{a_{1}}, \overline{a_{2}}\right) ;\left(\overline{b_{1}}, \overline{b_{2}}\right)\right]
\end{aligned}
$$

respectively calculate the upper and lower extremes of the zone of descendants of $\left(\overline{a_{1}}, \overline{a_{2}}\right)$ and $\left(\overline{b_{1}}, \overline{b_{2}}\right)$. And, in this case, the zone of descendants is the branch $d_{1} d_{2} \cdots d_{m}$. On the other hand,

$$
\begin{aligned}
& P H^{(i)}\left[\left(\overline{a_{1}}, \overline{a_{2}}\right) ;\left(\overline{b_{1}}, \overline{b_{2}}\right)\right] \text { and } \\
& P A^{(i)}\left[\left(\overline{a_{1}}, \overline{a_{2}}\right) ;\left(\overline{b_{1}}, \overline{b_{2}}\right)\right], \quad i=0,1,2,3, \cdots,
\end{aligned}
$$

respectively calculate the LAHCs in the increasing and decreasing directions of the branch $d_{1} d_{2} \cdots d_{m}$.

$3^{\text {rd }}$ case: $\left(\overline{b_{1}}, \overline{b_{2}}\right)$ is the third, fourth, ..., ancestor (except for the last one).

Let $\left(\overline{a_{1}}, \overline{a_{2}}\right)$ be the representative of a branch $d_{1} d_{2} \cdots d_{m}$ of $S_{i}$. Its ancestors in the periodic region are:

$$
\begin{aligned}
& \frac{1}{1} \cdot \frac{q_{1}}{p_{1}} \cdots \frac{q_{N-i}}{p_{N-i}}, \frac{1}{1} \cdot \frac{q_{1}}{p_{1}} \cdots \frac{q_{N-i+1}}{p_{N-i+1}}, \\
& \frac{1}{1} \cdot \frac{q_{1}}{p_{1}} \cdots \frac{q_{N-i+2}}{p_{N-i+2}}, \cdots, \frac{1}{1} \cdot \frac{q_{1}}{p_{1}} \cdots \frac{q_{N}}{p_{N}},
\end{aligned}
$$

and its ancestors in the chaotic region are the representatives of the branches $d_{1}, d_{1} d_{2}, \cdots, d_{1} d_{2} \cdots d_{m}$. Therefore, the third ancestor is $\frac{1}{1} \cdot \frac{q_{1}}{p_{1}} \cdots \frac{q_{N-i+2}}{p_{N-i+2}}$ if we are still in the periodic region, and the representative of the branch $d_{1}$ if we are not. Now,

$$
\begin{aligned}
& P H^{(\infty)}\left[\left(\overline{a_{1}}, \overline{a_{2}}\right) ;\left(\overline{b_{1}}, \overline{b_{2}}\right)\right] \text { and } \\
& P A^{(\infty)}\left[\left(\cdot \overline{a_{1}}, \overline{a_{2}}\right) ;\left(\overline{b_{1}}, \overline{b_{2}}\right)\right]
\end{aligned}
$$

respectively calculate the upper and lower extremes of the zone of descendants of $\left(\overline{a_{1}}, \overline{a_{2}}\right)$ and $\left(\overline{b_{1}}, \overline{b_{2}}\right)$, that in this case is a sub-branche of $d_{1} d_{2} \cdots d_{m}$. On the other hand,

$$
\begin{aligned}
& P H^{(i)}\left[\left(\overline{a_{1}}, \overline{a_{2}}\right) ;\left(\overline{b_{1}}, \overline{b_{2}}\right)\right] \text { and } \\
& P A^{(i)}\left[\left(\overline{a_{1}}, \overline{a_{2}}\right) ;\left(\overline{b_{1}}, \overline{b_{2}}\right)\right], \quad i=0,1,2,3, \cdots,
\end{aligned}
$$

respectively calculate the LAHCs in the increasing and decreasing directions of this sub-branch of $d_{1} d_{2} \cdots d_{m}$. 
$4^{\text {th }}$ case $:\left(\bar{b}, \overline{b_{2}}\right)=\left(\overline{a_{1}}, \overline{a_{2}}\right)$.

As was already seen, when $\left(\overline{b_{1}}, \overline{b_{2}}\right)=\left(\overline{a_{1}}, \overline{a_{2}}\right)$ Equations (5), (6), (7) and (8) become Equations (1), (2), (3) and (4), hence pseudoharmonics and pseudoantiharmonics become PRM harmonics and PRM antiharmonics. Therefore, in this case, by using pseudoharmonics and pseudoantiharmonics one can calculate the two extremes and the LAHCs of the midget associated to any cardioid, and alternatively the upper extreme and the LAHCs of the chaotic band associated to any disc.

\subsection{Applications}

Let us see an example for each of the four previous cases. Let us consider again the period- $5 \mathrm{HC}$

$\left(\overline{a_{1}}, \overline{a_{2}}\right)=(\overline{.00111}, \overline{.01000})$, that is the representative of the branch 11 of the shrub (1/3). We help us with Figure 5, which shows such a shrub (1/3), and with Figure 6 whose part (a) is a magnification of the branch 11, and whose part (b) is an additional magnification of the period-5 representative. If one starts from

$\left(\overline{a_{1}}, \overline{a_{2}}\right)=(\overline{.00111}, \overline{01000})$, the possible values of

$\left(\overline{b_{1}}, \overline{b_{2}}\right)$ are, as seen before: $(. \overline{0}, . \overline{1})$ and $(\overline{.001}, . \overline{010})$ in the periodic region, and $(\overline{.0011}, \overline{.0100})$ and $(\overline{.00111}, \overline{01000})$ in $S_{1}$, that is the only subshrub of the chaotic region.

\subsubsection{First Example}

Let us begin with $\left(\overline{b_{1}}, \overline{b_{2}}\right)=(. \overline{0}, . \overline{1})$, which corresponds to the $1^{\text {st }}$ case because $\left(\overline{b_{1}}, \overline{b_{2}}\right)$ is the generator of $S_{1}$, and therefore the first ancestor.

$$
P H^{(\infty)}[(\overline{00111}, \overline{01000}) ;(. \overline{0}, . \overline{1})]=(.00 \overline{1}, .01 \overline{0})
$$

is the Misiurewicz point $M_{2,1}$ placed in the upper extreme of the branch $111 \cdots$, ftip (1/3), that is the upper extreme of the increasing part of the zone of descendants of $[(\overline{.00111}, \overline{.01000}) ;(. \overline{0}, . \overline{1})]$, as can be clearly seen in

Figures 5(b), 5(c), and 6(a).

$$
\begin{aligned}
& P H^{(i)}[(\overline{.00111}, \overline{01000}) ;(. \overline{0}, . \overline{1})] \text { calculates } \\
& (\overline{.00111}, \overline{01000}),(\overline{.001111}, \overline{010000}), \\
& (\overline{.0011111}, \overline{.0100000}), \cdots
\end{aligned}
$$

which are the LAHCs in this increasing part of the zone of descendants, or, what is the same, the representatives of the branches 11, 111 and 1111 (the last one is not shown in the figure). In general, the increasing part of the zone of descendants starts from $\left(\overline{a_{1}}, \overline{a_{2}}\right)$, that is the representative of the branch $d_{1} d_{2} \cdots d_{m}$, and it reaches the upper extreme of its chaotic band by following the branches $d_{1} d_{2} \cdots d_{m}, d_{1} d_{2} \cdots d_{m} 1, \quad d_{1} d_{2} \cdots d_{m} 11, \cdots$. This is the only case in which pseudoantiharmonics can not be used in order to calculate the decreasing part of the zone of descendants of $[(\overline{.00111}, \overline{.01000}) ;(. \overline{0}, . \overline{1})]$.

\subsubsection{Second Example}

Let us go on with $\left(\overline{b_{1}}, \overline{b_{2}}\right)=(\overline{.001}, \overline{.010})$, that corresponds to the $2^{\text {nd }}$ case in which $\left(\overline{b_{1}}, \overline{b_{2}}\right)$ is the second ancestor, corresponding to $\frac{1}{1} \cdot \frac{q_{1}}{p_{1}} \cdots \frac{q_{N}}{p_{N}}$, that in this case is the last ancestor in the periodic region. The upper and lower extremes of the zone of descendants of

$$
\begin{aligned}
& {[(. \overline{00111}, \overline{.01000}) ;(. \overline{001}, \overline{.010})] \text { are }} \\
& \begin{aligned}
P H^{(\infty)} & {[(\overline{00111}, \overline{01000}) ;(\overline{.001}, \overline{.010})] } \\
\quad & (.00111 \overline{010}, .01000 \overline{001}) \text { and }
\end{aligned} \\
& P A^{(\infty)}[(\overline{.00111}, \overline{01000}) ;(\overline{.001}, \overline{.010})] \\
& \quad=(.0011 \overline{0}, .0100 \overline{001}),
\end{aligned}
$$

that are the Misiurewicz points $M_{5,1}$ and $M_{4,1}$, extremes of the branch 11 (see Figures 5(c) and 6(a)). As always,

$$
\begin{aligned}
& P H^{(i)}[(\overline{.00111}, \overline{.01000}) ;(\overline{.001}, \overline{.010})] \text { and } \\
& P A^{(i)}[(\overline{00111}, \overline{.01000}) ;(\overline{.001}, \overline{.010})]
\end{aligned}
$$

calculate the LAHCs in the increasing part $((\overline{.00111}, \overline{.01000}),(\overline{.00111010}, \overline{.01000001})$, $(\overline{.00111010010}, \overline{.01000001001}), \cdots)$ and in the decreasing part $((\overline{.00111}, \overline{01000}),(\overline{.00111001}, \overline{.01000010}))$, $(\overline{.00111001001}, \overline{.01000010010}), \cdots)$ of the zone of descendants of $[(\overline{.00111}, \overline{.01000}) ;(\overline{.001}, \overline{.010})]$, in this case the branch 11 .

\subsubsection{Third Example}

Let us go on with $\left(\overline{b_{1}}, \overline{b_{2}}\right)=(\overline{.0011}, \overline{0100})$ (the $3^{\text {rd }}$ case in which $\left(\overline{b_{1}}, \overline{b_{2}}\right)$ is the third ancestor). $(\overline{.0011}, \overline{.0100})$ is the representative of the branch 1, i.e. the first ancestor in the chaotic region $S_{1}$. The upper and lower extremes of the zone of descendants of 


$$
\begin{gathered}
{[(\overline{.00111}, \overline{.01000}) ;(\overline{.0011}, \overline{.0100})] \text { are }} \\
P H^{(\infty)}[(\overline{.00111}, \overline{01000}) ;(\overline{.0011}, \overline{.0100})] \\
=(.00111 \overline{0100}, .01000 \overline{0011}) \text { and } \\
P A^{(\infty)}[(\overline{.00111}, \overline{01000}) ;(\overline{.0011}, . \overline{0100})] \\
=(.0011100, .0100001),
\end{gathered}
$$

that are the Misiurewicz points $M_{5,2}$ and $M_{3,2}$, which are the extremes of a sub-branch of the branch 11 (see Figure 6(a)). This sub-branch is called $\operatorname{sub}_{1}\left(d_{1} d_{2} \cdots d_{m}\right)$. Again,

$$
\begin{aligned}
& P H^{(i)}[(\overline{00111}, \overline{01000}) ;(\overline{.0011}, . \overline{0100})] \text { and } \\
& P A^{(i)}[(\overline{.00111}, \overline{01000}) ;(.0011, .0100)]
\end{aligned}
$$

calculate the LAHCs in the increasing part

$((\overline{00111}, \overline{01000}),(\overline{.001110100}, \overline{010000011})$,

$(\overline{0011101000100}, \overline{0100000110011}), \cdots)$ and in the decreasing part $((\overline{.00111}, \overline{01000}),(\overline{.001110011}, \overline{010000100})$, $(\overline{0011100110011}, \overline{0100001000100}), \cdots)$ of the zone of descendants of such a sub-branch, $s u b_{1}\left(d_{1} d_{2} \cdots d_{m}\right)$, in both cases with periods $5,9,13, \cdots$, although only periods 5 and 9 are shown in the figure. If we were in a case where $\left(\bar{b}_{1}, \bar{b}_{2}\right)$ is the fourth ancestor (but not the last one), then $P H^{(\infty)}\left[\left(\overline{a_{1}}, \overline{a_{2}}\right) ;\left(\overline{b_{1}}, \overline{b_{2}}\right)\right]$ and $P A^{(\infty)}\left[\left(\overline{a_{1}}, \overline{a_{2}}\right) ;\left(\overline{b_{1}}, \overline{b_{2}}\right)\right]$ would be the upper and lower extremes of the zone of descendants of $\left[\left(\overline{a_{1}}, \overline{a_{2}}\right) ;\left(\overline{b_{1}}, \overline{b_{2}}\right)\right]$, which are the Misiurewicz points that are the extremes of a sub-branch $s u b_{2}\left(d_{1} d_{2} \cdots d_{m}\right)$ of the previous sub-branch $\operatorname{sub}_{1}\left(d_{1} d_{2} \cdots d_{m}\right)$. And so on.

\subsubsection{Fourth Example}

Finally, let us see the $\mathrm{n}^{\text {th }}$ case, that corresponds to $\left(\overline{b_{1}}, \overline{b_{2}}\right)=(\overline{.00111}, \overline{01000})$, i.e. when

$\left(\overline{b_{1}}, \overline{b_{2}}\right)=\left(\overline{a_{1}}, . \overline{a_{2}}\right)$. The upper and lower extremes of the zone of descendants of

$$
\begin{aligned}
& {[(\overline{.00111}, \overline{.01000}) ;(\overline{.00111}, \overline{.01000})] \text { are }} \\
& P H^{(\infty)}[(\overline{.00111}, \overline{01000}) ;(\overline{.00111}, \overline{01000})] \\
& \quad=(.00111 \overline{01000}, .01000 \overline{00111}) \text { and } \\
& P A^{(\infty)}[(\overline{.00111}, \overline{01000}) ;(\overline{.00111}, \overline{.01000})] \\
& \quad=(\overline{00111}, \overline{.01000}),
\end{aligned}
$$

that are the tip and the cusp of the midget associated to $\left(\overline{a_{1}}, \overline{a_{2}}\right)=\left(\overline{b_{1}}, \overline{b_{2}}\right)=(\overline{.00111}, \overline{01000})$, the first one a

Misiurewicz point $M_{5,5}$ and the second one the cusp of the original HC $\left(. \overline{a_{1}}, \overline{a_{2}}\right)=\left(\overline{b_{1}}, \overline{b_{2}}\right)=(\overline{.00111}, . \overline{01000})$ (see Figure 6(b)). As always, $P H^{(i)}[(\overline{.00111}, \overline{01000}) ;(\overline{.001}, \overline{010})]$ calculates the LAHCs of the increasing part of the zone of descendants $((\overline{.00111}, \overline{01000}),(\overline{.0011101000}, \overline{0100000111})$,

$(\overline{.001110100001000}, \overline{.010000011100111})$,

$(\overline{.00111010000100001000}, \overline{01000001110011100111}), \cdots)$.

This increasing part coincides with all the zone of descendants because the decreasing part of the zone of descendants becomes a point, the cusp of the original HC.

\section{Conclusions}

We review a branch of the harmonic analysis applied to dynamical systems. We focus on discrete dynamical systems, and more specifically on quadratic maps, both 1D quadratic maps and 2D quadratic maps, since these maps are the most widely used by experimental scientists, to whom we have addressed this paper.

We first review the applications in 1D quadratic maps, in particular the harmonics and antiharmonics introduced by MSS. Given a periodic orbit, the MSS harmonics of the symbolic sequence of this orbit calculate the symbolic sequences of the period doubling cascade of the orbit. Metropolis, Stein and Stein introduced for the first time this type of calculation, and the concept of harmonics in dynamical systems.

Based on MSS harmonics, Pastor, Romera and Montoya introduced the PRM harmonics. These type of harmonics expand the possibilities of the calculations. So, given the symbolic sequence of a periodic orbit, the PRM harmonics of this orbit are the symbolic sequences of the infinity of last appearance periodic orbits of the chaotic band where the original orbit is. Thus, if we start from the peiod- 1 orbit, we can calculate the structure of a 1D quadratic map.

Both, MSS harmonics and PRM harmonics are redefined in order to can be used in 2D quadratic maps where external arguments are used as identifiers instead of symbolic sequences. Given the external arguments of a cardioid, the MSS harmonics calculate the EAs of the discs of its period doubling cascade, and the PRM harmonics the EAs of its LAHCs of its chaotic band $B_{0}$.

Pseudoharmonics and pseudoantiharmonics, which are an expansion of PRM harmonics and PRM antiharmonics in the case of 2D quadratic maps, are also reviewed. These new calculation tools are notably more powerful than PRM harmonics and PRM antiharmonics and make possi- 
ble the calculation of LAHCs in new zones of descendants.

\section{Acknowledgments}

This work was partially supported by Ministerio de Ciencia e Innovación (Spain) under grant TIN 2011-22668.

\section{REFERENCES}

[1] P.-F. Verhulst, "Recherches Mathématiques sur la Loi d'Accroissement de la Population,” Mémoires de l'Académie Royale des Sciences et Belles-Lettres de Bruxelles, Bruxelles, 1844.

[2] S. Ulam and J. von Neumann, "On Combination of Stochastic and Deterministic Processes," Bulletin of the American Mathematical Society, Vol. 53, No. 11, 1947, p. 1120.

[3] R. M. May, "Simple Mathematical Models with Very Complicated Dynamics,” Nature, Vol. 261, 1976, pp. 459467. doi:10.1038/261459a0

[4] B. B. Mandelbrot, "Fractal Aspects of the Iteration of $z \rightarrow \lambda z(1-z)$ for Complex $\lambda$ and $z$," Annals of the New York Academy of Sciences, Vol. 357, 1980, pp. 249-259. doi:10.1111/j.1749-6632.1980.tb29690.x

[5] B. B. Mandelbrot, "On the Quadratic Mapping of $z \rightarrow z^{2}-\mu$ for Complex $\mu$ and $z$ : The Fractal Structure of Its $\mu$ Set, and Scaling," Physica D: Nonlinear Phenomena, Vol. 7, 1983, pp. 224-239. doi:10.1016/0167-2789(83)90128-8

[6] B. Branner, “The Mandelbrot Set," Proceedings of Symposia in Applied Mathematics, Vol. 39, 1989, pp. 75-105.

[7] J. Milnor and W. Thurston, "On Iterated Maps of the Interval,” Dynamical Systems, Vol. 1342, 1988, pp. 465563. doi:10.1007/BFb0082847

[8] H.-O. Peitgen, H. Jürgens and D. Saupe, "Chaos and Fractals,” Springer, New York, 1992, pp. 569-574.

[9] R. L. Devaney, “An Introduction to Chaotic Dynamical Systems,” Addison-Wesley, Boston, 1989, pp. 44-48.

[10] G. Pastor, M. Romera and F. Montoya, "An Approach to the Ordering of One-Dimensional Quadratic Maps,” Chaos, Solitons \& Fractals, Vol. 7, No. 4, 1996, pp. 565-584. doi:10.1016/0960-0779(95)00071-2

[11] M. Romera, G. Pastor, J. C. Sanz-Martín and F. Montoya, "Symbolic Sequences of One-Dimensional Quadratic Maps," Physica A: Statistical Mechanics and Its Applications, Vol. 256, No. 3-4, 1998, pp. 369-382. doi:10.1016/S0378-4371(98)00083-1

[12] G. Pastor, M. Romera and F. Montoya, "Harmonic Structure of One-Dimensional Quadratic Maps,” Physical Review E, Vol. 56, 1997, pp. 1476-1483. doi:10.1103/PhysRevE.56.1476

[13] J. Hale and H. Koçak, "Dynamics and Bifurcations," Applied Mathematics, Vol. 3, 1991, p. 314. doi:10.1007/978-1-4612-4426-4

[14] M. Misiurewicz and Z. Nitecki, "Combinatorial Patterns for Maps of the Interval," Memoirs of the American Mathematical Society, Vol. 94, No. 456, 1991, pp. 109-110.
[15] A. Douady and J. H. Hubbard, "Etude Dynamique des Polynômes Complexes,” Publications Mathematiques d’Orsay, 84-02, 1984 (Première Partie) and 85-04, 1985 (Deuxième Partie).

http://portail.mathdoc.fr/PMO/PDF/D_DOUADY_84_02. pdf

http://mathdoc.emath.fr/PMO/PDF/D_DOUADY_85_04. pdf

[16] M. Romera, G. Pastor and F. Montoya, "Misiurewicz Points in One-Dimensional Quadratic Maps,” Physica A: Statistical Mechanics and Its Applications, Vol. 232, No. 1-2, 1996, pp. 517-535. doi:10.1016/0378-4371(96)00127-6

[17] G. Pastor, M. Romera and F. Montoya, "On the Calculation of Misiurewicz Patterns in One-Dimensional Quadratic Maps," Physica A: Statistical Mechanics and Its Applications, Vol. 232, No. 1-2, 1996, pp. 536-553. doi:10.1016/0378-4371(96)00128-8

[18] G. Pastor, M. Romera, G. Alvarez and F. Montoya, "Misiurewicz Point Patterns Generation in One-Dimensional Quadratic Maps,” Physica A: Statistical Mechanics and Its Applications, Vol. 292, No. 1-4, 2001, pp. 207- 230. doi:10.1016/S0378-4371(00)00586-0

[19] G. Pastor, M. Romera, J. C. Sanz-Martín and F. Montoya, "Symbolic Sequences of One-Dimensional Quadratic Maps Points,” Physica A: Statistical Mechanics and Its Applications, Vol. 256, No. 3-4, 1998, pp. 369-382. doi:10.1016/S0378-4371(98)00083-1

[20] A. Douady and J. H. Hubbard, "Itération des Polynômes Quadratiques Complexes,” C. R. Academic Society: Série I, Paris, 1982.

[21] A. Douady, “Chaotic Dynamics and Fractals” Academic Press, New York, 1986.

[22] G. Pastor, M. Romera, G. Álvarez and F. Montoya, “Operating with External Arguments in the Mandelbrot Set Antenna,” Physica D: Nonlinear Phenomena, Vol. 117, No. 1-2, 2002, pp. 52-71. doi:10.1016/S0167-2789(02)00539-0

[23] N. Metropolis, M. L. Stein and P. R. Stein, "On Finite Limit Sets for Transformations on the Unit Interval," Journal of Combinatorial Theory: Series A, Vol. 15, No. 1, 1973, pp. 25-44. doi:10.1016/0097-3165(73)90033-2

[24] M. Morse and G. A. Hedlung, "Symbolic Dynamics," American Journal of Mathematics, Vol. 60, No. 4, 1938, pp. 815-866. doi:10.2307/2371264

[25] B.-L. Hao and W.-M. Zheng, "Symbolic Dynamics of Unimodal Maps Revisited,” International Journal of Modern Physics B, Vol. 3, No. 2, 1989, pp. 235-246. doi:10.1142/S0217979289000178

[26] M. Schroeder, "Fractals, Chaos, Power Laws," W. H. Freeman, New York, 1991.

[27] A. N. Sharkovsky, "Coexistence of Cycles of Continuous Mapping of the Line into Itself," Ukrainian Mathematical Journal, Vol. 16, 1964, pp. 61-71.

[28] A. N. Sharkovsky, Yu. L. Maistrenko and E. Yu. Romanenko, "Difference Equations and Their Applications," Kluver Academic Publishers, Dordrecht, 1993.

[29] M. Romera, G. Pastor and F. Montoya, "Graphic Tools to Analyse One-Dimensional Quadratic Maps,” Computer \& 
Graphics, Vol. 20, No. 2, 1996, pp. 333-339. doi:10.1016/0097-8493(95)00134-4

[30] M. Romera, G. Pastor and F. Montoya, "On the Cusp and the Tip of a Midget in the Mandelbrot Set Antenna," Physica Letters A, Vol. 221, No. 3, 1996, pp. 158-162.

[31] M. J. Feigenbaum, "Quantitative University for a Class of Nonlinear Transformations,” Journal of Statistical Physics, Vol. 19, No. 1, 1978, pp. 25-52. doi:10.1007/BF01020332

[32] R. L. Devaney, “Complex Dynamical Systems,” American Mathematical Society, Providence, 1994.

[33] M. Romera, G. Pastor, G. Alvarez and F. Montoya, "Shrubs in the Mandelbrot Set Ordering," International Journal of Bifurcation and Chaos, Vol. 13, No. 8, 2003, pp. 2279-2300. doi:10.1142/S0218127403007941

[34] G. Pastor, M. Romera, G. Alvarez and F. Montoya, “Ex- ternal Arguments for the Chaotic Bands Calculation in the Mandelbrot Set," Physica A: Statistical Mechanics and Its Applications, Vol. 353, No. 1, 2005, pp. 145-158. doi:10.1016/j.physa.2005.02.025

[35] G. Pastor, M. Romera, G. Alvarez, D. Arroyo, A. B. Orue, V. Fernández and F. Montoya, "A General View of Pseudoharmonics and Pseudoantiharmonics to Calculate External Arguments of Douady and Hubbard," Applied Mathematic and Computation, Vol. 213, No. 2, 2009, pp. 484-497. doi:10.1016/j.amc.2009.03.038

[36] G. Pastor, M. Romera, G. Alvarez, D. Arroyo and F. Montoya, "Equivalence between Subshrubs and Chaotic Bands in the Mandelbrot Set," Discrete Dynamics in Nature and Society, 2006, Article ID: 45920. doi:10.1155/DDNS/2006/70471 